\title{
Application of Artificial Intelligence for Nasopharyngeal Carcinoma Management - A Systematic Review
}

\author{
Wai Tong Ng, (D) ${ }^{1,2}$ Barton But, ${ }^{2}$ \\ Horace CW Choi, ${ }^{3}$ \\ Remco de Bree, ${ }^{4}$ Anne WM Lee, ${ }^{1,2}$ \\ Victor HF Lee, ${ }^{1,2}$ \\ Fernando López, 5,6 \\ Antti A Mäkitie, ${ }^{7-9}$ \\ Juan P Rodrigo, ${ }^{5,6}$ Nabil F Saba, (D) ${ }^{10}$ \\ Raymond KY Tsang, (D) ${ }^{11}$ \\ Alfio Ferlito $\mathbb{D}^{12}$ \\ 'Clinical Oncology Center, The University of \\ Hong Kong-Shenzhen Hospital, Shenzhen, \\ People's Republic of China; ${ }^{2}$ Department of \\ Clinical Oncology, Li Ka Shing Faculty of Medicine, \\ The University of Hong Kong, Hong Kong, China; \\ ${ }^{3}$ Department of Public Health, Li Ka Shing Faculty \\ of Medicine, The University of Hong Kong, Hong \\ Kong, China; ${ }^{4}$ Department of Head and Neck \\ Surgical Oncology, University Medical Center \\ Utrecht, Utrecht, the Netherlands; ${ }^{5}$ Department \\ of Otolaryngology, Hospital Universitario Central \\ de Asturias (HUCA), Instituto de Investigación \\ Sanitaria del Principado de Asturias (ISPA), \\ Instituto Universitario de Oncología del \\ Principado de Asturias (IUOPA), University of \\ Oviedo, Oviedo, 330II, Spain; ${ }^{6}$ Spanish \\ Biomedical Research Network Centre in \\ Oncology, CIBERONC, Madrid, 28029, Spain; \\ ${ }^{7}$ Department of Otorhinolaryngology - Head and \\ Neck Surgery, HUS Helsinki University Hospital \\ and University of Helsinki, Helsinki, Finland; \\ ${ }^{8}$ Research Program in Systems Oncology, Faculty \\ of Medicine, University of Helsinki, Helsinki, \\ Finland; 'Division of Ear, Nose and Throat \\ Diseases, Department of Clinical Sciences, \\ Intervention and Technology, Karolinska Institutet \\ and Karolinska University Hospital, Stockholm, \\ Sweden; ${ }^{10}$ Department of Hematology and \\ Medical Oncology, Emory University School of \\ Medicine, Atlanta, GA, USA; " 'Division of \\ Otorhinolaryngology, Department of Surgery, Li \\ Ka Shing Faculty of Medicine, The University of \\ Hong Kong, Hong Kong, People's Republic of \\ China; ${ }^{12}$ Coordinator of the International Head \\ and Neck Scientific Group, Padua, Italy
}

Correspondence: Barton But

Department of Clinical Oncology, Li Ka

Shing Faculty of Medicine, The University

of Hong Kong, Hong Kong, People's

Republic of China

Tel +852 22554352

Fax +85228726426

Email bbut@hku.hk
Introduction: Nasopharyngeal carcinoma (NPC) is endemic to Eastern and South-Eastern Asia, and, in 2020, 77\% of global cases were diagnosed in these regions. Apart from its distinct epidemiology, the natural behavior, treatment, and prognosis are different from other head and neck cancers. With the growing trend of artificial intelligence (AI), especially deep learning (DL), in head and neck cancer care, we sought to explore the unique clinical application and implementation direction of AI in the management of NPC.

Methods: The search protocol was performed to collect publications using AI, machine learning (ML) and DL in NPC management from PubMed, Scopus and Embase. The articles were filtered using inclusion and exclusion criteria, and the quality of the papers was assessed. Data were extracted from the finalized articles.

Results: A total of 78 articles were reviewed after removing duplicates and papers that did not meet the inclusion and exclusion criteria. After quality assessment, 60 papers were included in the current study. There were four main types of applications, which were auto-contouring, diagnosis, prognosis, and miscellaneous applications (especially on radiotherapy planning). The different forms of convolutional neural networks (CNNs) accounted for the majority of DL algorithms used, while the artificial neural network (ANN) was the most frequent ML model implemented.

Conclusion: There is an overall positive impact identified from AI implementation in the management of NPC. With improving AI algorithms, we envisage AI will be available as a routine application in a clinical setting soon.

Keywords: machine learning, neural network, deep learning, prognosis, diagnosis, auto contouring

\section{Introduction}

According to the International Agency for Research on Cancer, nasopharyngeal carcinoma (NPC) is the twenty-third most common cancer worldwide. The global number of new cases and deaths in 2020 were 133,354 and 80,008, respectively. ${ }^{1,2}$ Although it is not uncommon, it has a distinct geographical distribution where it is most prevalent in Eastern and South-Eastern Asia, accounting for 76.9\% of global cases. It was also found that almost half of the new cases occurred in China. ${ }^{2}$ Because of its late symptoms and anatomical location, it makes it difficult to be detected in the early stages. Radiotherapy is the primary treatment modality, and concomitant/adjunctive chemotherapy is often needed for advanced locoregional

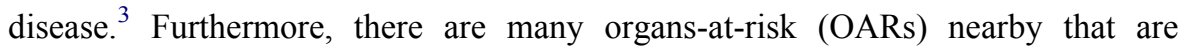


sensitive to radiation; these include the salivary glands, brainstem, optic nerves, temporal lobes and the cochlea. ${ }^{4}$ Hence, it is of interest whether the use of artificial intelligence (AI) can help improve the diagnosis, treatment process and prediction of outcomes for NPC.

With the advances of AI over the past decade, it has become pervasive in many industries playing both major and minor roles. This includes cancer treatment, where medical professionals search for methods to utilize it to improve treatment quality. AI refers to any method that allows algorithms to mimic intelligent behavior. It has two subsets, which are machine learning (ML) and deep learning (DL). ML uses statistical methods to allow the algorithm to learn and improve its performance, such as random forest and support vector machine. Artificial neural network (ANN) is an example of ML and is also a core part of DL. ${ }^{5}$ DL can be defined as a learning algorithm that can automatically update its parameters through multiple layers of ANN. Deep neural networks such as convolutional neural network $(\mathrm{CNN})$ and recurrent neural network are all DL architectures.

Besides histological, clinical and demographic information, a wide range of data ranging from genomics, proteomics, immunohistochemistry, and imaging must be integrated by physicians when developing personalized treatment plans for patients. This has led to an interest in developing computational approaches to improve medical management by providing insights that will enhance patient outcomes and workflow throughout a patient's journey.

Given the increased use of AI in cancer care, in this systematic literature review, papers on $\mathrm{AI}$ applications for NPC management were compiled and studied in order to provide an overview of the current trend. Furthermore, possible limitations discussed within the articles were explored.

\section{Methods}

\section{Search Protocol}

A systematic literature search was conducted to retrieve all studies that used AI or its subfields in NPC management. Keywords were developed and combined using boolean logic to produce the resulting search phrase: ("artificial intelligence" OR "machine learning" OR "deep learning" OR "Neural Network") AND ("nasopharyngeal carcinoma" OR "nasopharyngeal cancer"). Using the search phrase, a search of research articles from the past 15 years to March 2021 was performed on PubMed, Scopus and Embase. The results from the three databases were consolidated, and duplicates were removed. The Preferred Reporting Items for Systematic Review and MetaAnalyses (PRISMA) was followed where possible, and the PRISMA flow diagram and checklist were used as a guidelines to consider the key aspects of a systematic literature review. ${ }^{6}$

\section{Eligibility}

Exclusion and inclusion criteria were determined to assess the eligibility of the retrieved publications. The articles were first checked to remove those that were not within the exclusion criteria. These included book chapters, conference reports, literature reviews, editorials, letters to the editors and case reports. In addition, articles in languages other than English or Chinese and papers with inaccessible full-texts were also excluded.

The remaining studies were then filtered by reading the title and abstract to remove any articles that were not within the inclusion criteria (applications of AI or its subfield and experiments on NPC). A full-text review was further performed to confirm the eligibility of the articles based on both these criteria. The process was conducted by two independent reviewers (B.B \& H.C.).

\section{Data Extraction}

Essential information from each article was extracted and placed in a data extraction table (Table 1). These included the author(s), year of publication, country, sample type, sample size, AI algorithm used, application type, study aim, performance metrics reported, results, conclusion, and limitations. The AI model with the best performance metrics from each study was selected and included. Moreover, the performance results of models trained with the training cohort were obtained from evaluating the test cohort instead of the training cohort. This was to prevent overfitting by avoiding to train and test models with the same dataset.

\section{Quality Assessment}

The selected articles were assessed for risk of bias and applicability using the Quality Assessment of Diagnostic Accuracy Studies (QUADAS)-2 tool in Table 2. ${ }^{7}$ Studies with more than one section rated "high" or "unclear" were eliminated. Further quality assessment was also completed to ensure the papers meet the required standard. This was performed using the guidelines for developing and 


\begin{tabular}{|c|c|c|c|c|c|c|c|c|}
\hline $\begin{array}{l}\text { Authors, } \\
\text { Year and } \\
\text { Country }\end{array}$ & $\begin{array}{l}\text { Site, No. of Cases } \\
\text { (Data Type) }\end{array}$ & $\begin{array}{l}\text { Al Subfield } \\
\text { (Application) }\end{array}$ & $\begin{array}{c}\text { Artificial } \\
\text { Intelligence } \\
\text { Methods and its } \\
\text { Application }\end{array}$ & Study Aim & Performance Metric (s) & Results & Conclusion & Limitations \\
\hline $\begin{array}{l}\text { Wang e tal } \\
\left(20100^{\prime 2} \text { (Hong }\right. \\
\text { Kong SAR, } \\
\text { China) }\end{array}$ & $\begin{array}{c}\text { NPC and other type } \\
54 \\
\text { (Protein data) }\end{array}$ & $\begin{array}{l}\text { Machine } \\
\text { learning } \\
\text { (Diagnosis) }\end{array}$ & $\begin{array}{l}\text { I. Classification: } \\
\text { Non-linear } \\
\text { regulatory network } \\
\text { (hopfield-like } \\
\text { network) }\end{array}$ & $\begin{array}{l}\text { To find relationships } \\
\text { between protein } \\
\text { biomarkers and classify } \\
\text { different disease groups }\end{array}$ & $\begin{array}{l}\text { I. Classification performance } \\
\text { - Sensitivity } 0.95 \\
\text { - Specificity } 0.80 \\
\text { - Accuracy: } 0.93\end{array}$ & $\begin{array}{l}\text { The developed regulatary network out-performed } \\
\text { Fisher linear discriminant, KNN, linear SVM and } \\
\text { radial basis function SVM in classification } \\
\text { performance. }\end{array}$ & $\begin{array}{l}\text { The proposed technique has } \\
\text { promise in assisting disease } \\
\text { diagnosis by finding protein } \\
\text { regulation relationships. }\end{array}$ & N/A \\
\hline $\begin{array}{l}\text { Aussem et al } \\
(2012)^{11} \\
\text { (France) }\end{array}$ & $\begin{array}{l}\text { NPC } \\
\quad 1298 \\
\text { (Risk factors) }\end{array}$ & 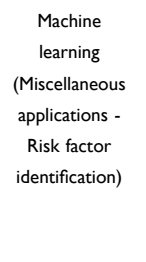 & $\begin{array}{l}\text { 1. Feature } \\
\text { selection: Markov } \\
\text { boundary discovery } \\
\text { algorithm } \\
\text { 2. lassification: } \\
\text { Bayesian network }\end{array}$ & $\begin{array}{l}\text { To extract relevant } \\
\text { dietary, social and } \\
\text { environmental risk } \\
\text { factors related to } \\
\text { increasing risk of NPC }\end{array}$ & $\begin{array}{l}\text { 1. Identification of potential risk } \\
\text { factors } \\
\text { - Odd ratio } \\
\text { 2. Predictive performance } \\
\text { - AUC: } 0.743 \\
\text { 3. Empirial performance } \\
\text { - Accuracy: } 0.654 \\
\text { - Euclidean distance }\end{array}$ & $\begin{array}{l}\text { The proposed model had a better performance in } \\
\text { recognizing risk factors associated with NPC than } \\
\text { other algorithms. }\end{array}$ & $\begin{array}{l}\text { The proposed techniques can } \\
\text { integrate experts' knowledge and } \\
\text { information extracted from data to } \\
\text { analyze epidemiologic data }\end{array}$ & N/A \\
\hline $\begin{array}{l}\text { Kumdee, } \\
\text { Bhongmakapat, } \\
\text { and Ritthipravat } \\
(2012)^{12} \\
\text { (Thailand) }\end{array}$ & $\begin{array}{c}\text { NPC } \\
\text { 1257 } \\
\text { (Clinicopathological } \\
\text { and serology data) }\end{array}$ & $\begin{array}{l}\text { Machine } \\
\text { learning } \\
\text { (Prognosis) }\end{array}$ & $\begin{array}{l}\text { 1. Prediction: } \\
\text { Generalized neural } \\
\text { network-type SIRM } \\
\text { (G-NN-SIRM) }\end{array}$ & $\begin{array}{l}\text { To predict NPC } \\
\text { recurrence }\end{array}$ & $\begin{array}{l}\text { 1. Classification performance } \\
\text { - AUC: } 0.8809 \\
\text { - Sensitivity: } 0.8003 \\
\text { - Specificty: } 0.8486 \\
\text { - Accuracy: } 0.8245 \\
\text { 2. Predicive erformance } \\
\text { - Mean square errror: } 0.595360\end{array}$ & $\begin{array}{l}\text { G-NN-SIRM had a significantly higher performance } \\
\text { than the other techniques in NPC recurrence } \\
\text { prediction }\end{array}$ & $\begin{array}{l}\text { The G-NN-SIRM can be applied to } \\
\text { NPC recurrence prediction }\end{array}$ & N/A \\
\hline $\begin{array}{l}\text { Ritthipravat, } \\
\text { Kumdee, and } \\
\text { Bhongmakapat } \\
(2013)^{13} \\
\text { (Thailand) }\end{array}$ & $\begin{array}{c}\text { NPC } \\
495 \\
\text { (Clinicopathological } \\
\text { and serology data) }\end{array}$ & $\begin{array}{c}\text { Machine } \\
\text { learning } \\
\text { (Prognosis) }\end{array}$ & $\begin{array}{l}\text { 1. Missing data } \\
\text { technique to } \\
\text { complete data for } \\
\text { model training: } \\
\text { Commlete-case } \\
\text { analysis, Mean } \\
\text { imputation, } \\
\text { expectation- } \\
\text { maximization } \\
\text { imputation and } \\
\text { KNN inputation } \\
\text { 2. Prognosis: } \\
\text { Siggle-point ANN } \\
\text { model, Multiple- } \\
\text { point ANN model } \\
\text { and Sequential } \\
\text { neural network }\end{array}$ & $\begin{array}{l}\text { To predict NPC } \\
\text { recurrence }\end{array}$ & $\begin{array}{l}\text { I. Predictive performance } \\
\text { - AUC ( } 5 \text { years): } 0.7300 \\
\text { - Chi-square statistics of goodness of } \\
\text { fit test (5 years recurrence-free } \\
\text { survival): } 4.30\end{array}$ & $\begin{array}{l}\text { The closest performance to the Kaplan-Meier } \\
\text { model were the expectation-maximization } \\
\text { imputataion technique models, particularly with } \\
\text { sequential neural network. }\end{array}$ & $\begin{array}{l}\text { Missing data technique cross- } \\
\text { combined with ANNs were } \\
\text { investigated for predicting NPC } \\
\text { recurrence. }\end{array}$ & N/A \\
\hline
\end{tabular}


Table I (Continued).

\begin{tabular}{|c|c|c|c|c|c|c|c|c|}
\hline $\begin{array}{l}\text { Authors, } \\
\text { Year and } \\
\text { Country }\end{array}$ & $\begin{array}{l}\text { Site, No. of Cases } \\
\text { (Data Type) }\end{array}$ & $\begin{array}{l}\text { Al Subfield } \\
\text { (Application) }\end{array}$ & $\begin{array}{l}\text { Artificial } \\
\text { Intelligence } \\
\text { Methods and its } \\
\text { Application }\end{array}$ & Study Aim & Performance Metric (s) & Results & Conclusion & Limitations \\
\hline $\begin{array}{l}\text { Zhu, and Kan } \\
(2014)^{44} \\
\text { (China) }\end{array}$ & $\begin{array}{c}\text { NPC } \\
312 \\
\text { (microRNA } \\
\text { expressions data) }\end{array}$ & $\begin{array}{l}\text { Machine } \\
\text { learning } \\
\text { (Prognosis) }\end{array}$ & $\begin{array}{l}\text { 1. Data } \\
\text { transformation, } \\
\text { data integration, or } \\
\text { prediction output: } \\
\text { ANN } \\
\text { 2. Death risk } \\
\text { assessment: } \\
\text { Neural network } \\
\text { cascade }\end{array}$ & $\begin{array}{l}\text { To assess cancer } \\
\text { prognosis }\end{array}$ & $\begin{array}{l}\text { I. Risk prediction performance } \\
\text { - AUC: } 0.95 \text { I } \\
\text { - Accuracy: } 0.83\end{array}$ & $\begin{array}{l}\text { The neural network cascade out-performed both } \\
\text { the transformed and untransformed neural } \\
\text { network models. }\end{array}$ & $\begin{array}{l}\text { The study proposed a potential } \\
\text { method for constructing a } \\
\text { microRNA biomarker selection and } \\
\text { prediction model }\end{array}$ & N/A \\
\hline $\begin{array}{l}\text { Jiang et al. } \\
(2016)^{14} \\
\text { (China) }\end{array}$ & $\begin{array}{c}\text { NPC } \\
453 \\
\text { (Clinicopathological } \\
\text { and serology data) }\end{array}$ & $\begin{array}{l}\text { Machine } \\
\text { learning } \\
\text { (Prognosis) }\end{array}$ & $\begin{array}{l}\text { I. Feature } \\
\text { Selection: } \\
\text { Recursive feature } \\
\text { elimination } \\
\text { procedure based } \\
\text { on SVM } \\
\text { 2. Classification: } \\
\text { SVM }\end{array}$ & $\begin{array}{l}\text { To predict the survival } \\
\text { of NPC patients with } \\
\text { synchronous } \\
\text { metastases }\end{array}$ & $\begin{array}{l}\text { I. Prognostic performance } \\
\text { - AUC: } 0.633 \\
\text { - Sensitivity: } 0.713 \\
\text { - Specificity: } 0.807\end{array}$ & $\begin{array}{l}\text { The ML model had a better prognostic } \\
\text { performance than classifiers using clinical indexes } \\
\text { alone or with haematological markers }\end{array}$ & $\begin{array}{l}\text { The model has the potential to help } \\
\text { clinicians choose the most } \\
\text { appropriate treatment strategy for } \\
\text { metastatic NPC patients }\end{array}$ & $\begin{array}{l}\text { I. Not all clinical indexes and } \\
\text { haematological markers were } \\
\text { included in the study. } \\
\text { 2. The effectiveness of the } \\
\text { combination treatment is } \\
\text { uncertain due to differences in } \\
\text { being treated in different } \\
\text { institutions }\end{array}$ \\
\hline $\begin{array}{l}\text { Liu et al } \\
(2016)^{43} \\
\text { (China) }\end{array}$ & $\begin{array}{c}\text { NPC } \\
53 \\
\text { (MRI images) }\end{array}$ & $\begin{array}{l}\text { Machine } \\
\text { learning } \\
\text { (Prognosis) }\end{array}$ & $\begin{array}{l}\text { I. Classification: } \\
\text { KNN \& ANN }\end{array}$ & $\begin{array}{l}\text { To predict NPC } \\
\text { response to } \\
\text { chemoradiotherapy }\end{array}$ & $\begin{array}{l}\text { I. Classification performance } \\
\text { - Specificity: } 1.000 \\
\text { - Accuracy: } 0.909 \\
\text { - Precision: } 1.000 \\
\text { - F-score: } 0.875 \\
\text { - Matthews correlation coefficient: } \\
0.810 \\
\text { - No information rate: } 0.727\end{array}$ & $\begin{array}{l}\text { The model using parameters extracted from } \mathrm{TI} \\
\text { sequence had a better classification performance } \\
\text { than the other two models }\end{array}$ & $\begin{array}{l}\text { Integrating texture parameters to } \\
\text { ML algorithms can act as imaging } \\
\text { biomarkers for NPC tumor } \\
\text { response to chemoradiotherapy }\end{array}$ & I. Relatively small sample size \\
\hline $\begin{array}{l}\text { Wang et al } \\
(2016)^{63} \\
\text { (Taiwan and } \\
\text { China) }\end{array}$ & $\begin{array}{l}\text { NPC } \\
335 \\
\text { (MRI and PET/CT } \\
\text { images) }\end{array}$ & $\begin{array}{l}\text { Machine } \\
\text { learning } \\
\text { (Diagnosis) }\end{array}$ & $\begin{array}{l}\text { I. Classification: } \\
\text { ANN }\end{array}$ & $\begin{array}{l}\text { To assess the diagnostic } \\
\text { accuracy of adding } \\
\text { additional nodal } \\
\text { parameters and PET/ } \\
\text { CT }\end{array}$ & $\begin{array}{l}\text { I. Diagnostic efficacy } \\
\text { - Sensitivity: } 0.822 \\
\text { - Specificity: } 0.952 \\
\text { - Youden's index } \\
\text { - Accuracy: } 0.888 \\
\text { - PPV: } 0.943 \\
\text { - NPV: } 0.846\end{array}$ & $\begin{array}{l}\text { ANN demonstrated that combining three (and } \\
\text { four) of the proposed parameters yielded good } \\
\text { results. }\end{array}$ & $\begin{array}{l}\text { Additional parameters in MRI and } \\
\text { PET/CT were found which can } \\
\text { improve prediction accuracy. }\end{array}$ & $\begin{array}{l}\text { 1. Possible diagnostic errors from } \\
\text { not using histopathology. } \\
\text { 2. Not all factors useful in the } \\
\text { diagnosis were included due to } \\
\text { limitations of the facility in the } \\
\text { center. }\end{array}$ \\
\hline
\end{tabular}




\begin{tabular}{|c|c|c|c|c|c|c|c|c|}
\hline $\begin{array}{l}\text { Men et al } \\
(2017)^{55} \\
\text { (China) }\end{array}$ & $\begin{array}{c}\text { NPC } \\
230 \\
\text { (CT images) }\end{array}$ & $\begin{array}{l}\text { Deep learning } \\
\text { (Auto- } \\
\text { contouring) }\end{array}$ & $\begin{array}{l}\text { I. Segmentation: } \\
\text { Deep } \\
\text { deconvolutional } \\
\text { neural network }\end{array}$ & $\begin{array}{l}\text { To assess the } \\
\text { segmentation ability of } \\
\text { the developed model }\end{array}$ & $\begin{array}{l}\text { I. Segmentation performance } \\
\text { - DSC: } 0.753 \pm 0.113 \\
\text { - HD: } 12.6 \mathrm{~mm} \pm 11.5 \mathrm{~mm}\end{array}$ & $\begin{array}{l}\text { The deep deconvolutional neural network had a } \\
\text { much better performance when compared with } \\
\text { VGG-16 model. }\end{array}$ & $\begin{array}{l}\text { The proposed model has promise in } \\
\text { enhancing the consistency of } \\
\text { delineation and streamline the } \\
\text { radiotherapy workflows. }\end{array}$ & $\begin{array}{l}\text { I. The model may be hard to } \\
\text { converge due to having } \mathrm{NO} \text { and } \mathrm{N} \\
\text { + patients in both the training and } \\
\text { testing set. } \\
\text { 2. Interobserver variability may } \\
\text { cause bias } \\
\text { 3. The segmentation performance } \\
\text { may be affected by the inclusion of } \\
\text { both stage I and II patients as the } \\
\text { target may have diverse contrast, } \\
\text { shape and sizes. }\end{array}$ \\
\hline $\begin{array}{l}\text { Mohammed } \\
\text { et al }(2017)^{54} \\
\text { (Malaysia and } \\
\text { Iraq) }\end{array}$ & $\begin{array}{c}\text { NPC } \\
149 \\
\text { (Microscopic } \\
\text { images) }\end{array}$ & $\begin{array}{l}\text { Machine } \\
\text { learning (Auto- } \\
\text { contouring/ } \\
\text { Diagnosis) }\end{array}$ & $\begin{array}{l}\text { I. Segmentation: } \\
\text { K-means clustering } \\
\text { algorithm and ANN } \\
\text { 2. Classification: } \\
\text { K-means clustering } \\
\text { algorithm and ANN }\end{array}$ & $\begin{array}{l}\text { To evaluate the } \\
\text { segmentation and } \\
\text { identification } \\
\text { performance of the } \\
\text { developed models }\end{array}$ & $\begin{array}{l}\text { I. Classification performance } \\
\text { - Sensitivity: } 0.9324 \\
\text { - Specificity: } 0.9054 \\
\text { - Accuracy: } 0.911 \\
\text { 2. Segmentation performance } \\
\text { - Accuracy: } 0.883\end{array}$ & $\begin{array}{l}\text { The proposed method had an improved } \\
\text { classification and segmentation performance over } \\
\text { SVM. }\end{array}$ & $\begin{array}{l}\text { Texture features can assist in } \\
\text { differentiating benign and malignant } \\
\text { tumors. Thus, the fully automated } \\
\text { proposed model can help with } \\
\text { doctors' diagnosis and support } \\
\text { them. }\end{array}$ & $\begin{array}{l}\text { 1. Disproportionate distribution } \\
\text { between benign and malignant } \\
\text { tumors in the dataset. } \\
\text { 2. Sample size can be larger to } \\
\text { improve reliability } \\
\text { 3. Combining different techniques } \\
\text { is complicated. }\end{array}$ \\
\hline $\begin{array}{l}\text { Zhang et al } \\
(2017)^{42} \\
\text { (China) }\end{array}$ & $\begin{array}{c}\text { NPC } \\
110 \\
\text { (MRI images) }\end{array}$ & $\begin{array}{l}\text { Machine } \\
\text { learning } \\
\text { (Prognosis) }\end{array}$ & $\begin{array}{l}\text { I. Classification: } \\
\text { LR, Kernel SVM, } \\
\text { Linear SVM, } \\
\text { AdaBoost, RF, } \\
\text { ANN, KNN, Linear } \\
\text { discriminant } \\
\text { analysis and Naiive } \\
\text { bayes } \\
\text { 2. Feature } \\
\text { Selection: } \\
\text { LR, Logistic SVM, } \\
\text { RF, Distance } \\
\text { correlation, Elastic } \\
\text { net LR \& Sure } \\
\text { independence } \\
\text { screening }\end{array}$ & $\begin{array}{l}\text { To predict local and } \\
\text { distant failure of } \\
\text { advanced NPC } \\
\text { patients' prior } \\
\text { treatment }\end{array}$ & $\begin{array}{l}\text { I. Prognostic performance } \\
\text { - AUC: } 0.8464 \pm 0.0069 \\
\text { - Test error: } 0.3135 \pm 0.0088\end{array}$ & $\begin{array}{l}\text { Using RF for both feature selection and } \\
\text { classification had the best prognostic performance }\end{array}$ & $\begin{array}{l}\text { The most optimal ML methods for } \\
\text { local and distant failure prediction in } \\
\text { advanced NPC can improve } \\
\text { precision oncology and clinical } \\
\text { practice }\end{array}$ & $N / A$ \\
\hline $\begin{array}{l}\text { Zhang et al } \\
(2017)^{41} \\
\text { (China) }\end{array}$ & $\begin{array}{c}\text { NPC } \\
118 \\
\text { (MRI images) }\end{array}$ & $\begin{array}{l}\text { Machine } \\
\text { learning } \\
\text { (Prognosis) }\end{array}$ & $\begin{array}{l}\text { I. Analysis } \\
\text { Clustering (with } \\
\text { radiomics) }\end{array}$ & $\begin{array}{l}\text { Individualized } \\
\text { progression-free } \\
\text { survival evaluation of } \\
\text { advanced NPC } \\
\text { patients' prior } \\
\text { treatment }\end{array}$ & $\begin{array}{l}\text { I. Prognostic performance } \\
\text { - C-index: } 0.776 \text { [Cl: } 0.678-0.873]\end{array}$ & $\begin{array}{l}\text { Integrating radiomics signature with other factors } \\
\text { within a nomogram such as TNM staging system or } \\
\text { clinical data improved its performance. }\end{array}$ & $\begin{array}{l}\text { The use of quantitative radiomics } \\
\text { models can be useful in precision } \\
\text { medicine and assist with the } \\
\text { treatment strategies for NPC } \\
\text { patients }\end{array}$ & $\begin{array}{l}\text { 1. The analysis did not consider } \\
\text { two-way or higher order } \\
\text { interactions between features. } \\
\text { 2. The generalizability of the } \\
\text { model cannot be determined as } \\
\text { the validation cohort was taken } \\
\text { from the same institution. } \\
\text { 3. The training phase of the model } \\
\text { may be affected from selection } \\
\text { bias due to stringent inclusion } \\
\text { criteria. }\end{array}$ \\
\hline
\end{tabular}

(Continued) 
Table I (Continued).

\begin{tabular}{|c|c|c|c|c|c|c|c|c|}
\hline $\begin{array}{l}\text { Authors, } \\
\text { Year and } \\
\text { Country }\end{array}$ & $\begin{array}{l}\text { Site, No. of Cases } \\
\text { (Data Type) }\end{array}$ & $\begin{array}{l}\text { Al Subfield } \\
\text { (Application) }\end{array}$ & $\begin{array}{l}\text { Artificial } \\
\text { Intelligence } \\
\text { Methods and its } \\
\text { Application }\end{array}$ & Study Aim & Performance Metric (s) & Results & Conclusion & Limitations \\
\hline $\begin{array}{l}\text { Li et al }(2018)^{15} \\
\text { (China) }^{15}\end{array}$ & $\begin{array}{c}\text { NPC } \\
30396 \\
\text { (Endoscopic images) }\end{array}$ & $\begin{array}{l}\text { Deep learning } \\
\quad \text { (Auto- } \\
\text { contouring/ } \\
\text { Diagnosis) }\end{array}$ & $\begin{array}{l}\text { I. Detection: Fully } \\
\text { CNN }\end{array}$ & $\begin{array}{l}\text { To evaluate the } \\
\text { performance of the } \\
\text { developed model to } \\
\text { segment and detect } \\
\text { nasopharyngeal } \\
\text { malignancies in } \\
\text { endoscopic images }\end{array}$ & $\begin{array}{l}\text { I. Detection performance } \\
\text { - AUC: } 0.930 \text { - Sensitivity: } 0.902 \text { [Cl: } \\
0.878-0.922] \\
\text { - Specificity: } 0.855 \text { [Cl: } 0.827-0.880] \\
\text { - Accuracy: } 0.880 \text { [Cl: } 0.861-0.896] \\
\text { - PPV: } 0.869 \text { [Cl: } 0.843-0.892] \\
\text { - NPV: } 0.892 \text { [CI: } 0.865-0.914] \\
\text { - Time taken: } 0.67 \text { min } \\
\text { 2. Segmentation performance } \\
\text { - DSC: } 0.75 \pm 0.26\end{array}$ & $\begin{array}{l}\text { The developed model had a better performance } \\
\text { than oncologists in nasopharyngeal mass } \\
\text { differentiation. } \\
\text { It was also able to automatically segment malignant } \\
\text { areas from the background of nasopharyngeal } \\
\text { endoscopic images. }\end{array}$ & $\begin{array}{l}\text { The proposed method has potential } \\
\text { in guided biopsy for nasopharyngeal } \\
\text { malignancies. }\end{array}$ & $\begin{array}{l}\text { I. Limited diversity due to all } \\
\text { samples being acquired from the } \\
\text { same institution, which leads to } \\
\text { over-fitting. }\end{array}$ \\
\hline $\begin{array}{l}\text { Mohammed } \\
\text { et al }(2018)^{62} \\
\text { (Malaysia, Iraq } \\
\text { and India) }\end{array}$ & $\begin{array}{c}\text { NPC } \\
381 \\
\text { (Endoscopic images) }\end{array}$ & $\begin{array}{l}\text { Artificial } \\
\text { intelligence and } \\
\text { machine } \\
\text { learning } \\
\text { (Diagnosis) }\end{array}$ & $\begin{array}{l}\text { 1. Feature } \\
\text { selection: } \\
\text { Genetic algorithm } \\
\text { 2. Classification: } \\
\text { ANN }\end{array}$ & $\begin{array}{l}\text { To evaluate the } \\
\text { proposed method in } \\
\text { detecting NPC from } \\
\text { endoscopic images }\end{array}$ & $\begin{array}{l}\text { I. Classification performance } \\
\text { - Sensitivity: } 0.9535 \\
\text { - Specificity: } 0.9455 \\
\text { - Accuracy: } 0.9622\end{array}$ & $\begin{array}{l}\text { The detection performance of the trained ANN } \\
\text { was close to that done manually by ear, nose and } \\
\text { throat specialists. }\end{array}$ & $\begin{array}{l}\text { The study demonstrated the } \\
\text { feasibility of using ANNs for NPC } \\
\text { identification in endoscopic images. }\end{array}$ & N/A \\
\hline $\begin{array}{l}\text { Mohammed } \\
\text { et al }(2018)^{16} \\
\text { (Malaysia, Iraq } \\
\text { and India) }\end{array}$ & $\begin{array}{c}\text { NPC } \\
381 \\
\text { (Endoscopic images) }\end{array}$ & $\begin{array}{l}\text { Artificial } \\
\text { intelligence and } \\
\text { machine } \\
\text { learning (Auto- } \\
\text { contouring/ } \\
\text { Diagnosis) }\end{array}$ & $\begin{array}{l}\text { I. Feature } \\
\text { selection: } \\
\text { Genetic algorithm } \\
\text { 2. Classification: } \\
\text { ANN \& SVM }\end{array}$ & $\begin{array}{l}\text { To evaluate the } \\
\text { proposed model in } \\
\text { diagnosing NPC from } \\
\text { endoscopic images }\end{array}$ & $\begin{array}{l}\text { 1. Segmentation performance } \\
\text { - Accuracy: } 0.9265 \\
\text { 2. Classification performance } \\
\text { - Sensitivity: } 0.9480 \\
\text { - Specificity: } 0.9520 \\
\text { - Precision: } 0.9515\end{array}$ & $\begin{array}{l}\text { The developed model yielded similar results to that } \\
\text { of ENT specialists in segmentation performance. } \\
\text { The classification performance achieved high results } \\
\text { but the training dataset had a better performance. }\end{array}$ & $\begin{array}{l}\text { The study demonstrated the } \\
\text { effectiveness and accuracy of the } \\
\text { proposed method. }\end{array}$ & N/A \\
\hline $\begin{array}{l}\text { Du et al } \\
(2019)^{40} \text { (Hong } \\
\text { Kong SAR, } \\
\text { China) }\end{array}$ & $\begin{array}{l}\text { NPC } \\
277 \\
\text { (MRI images and } \\
\text { clinicopathological } \\
\text { data) }\end{array}$ & $\begin{array}{l}\text { Machine } \\
\text { learning } \\
\text { (Prognosis) }\end{array}$ & $\begin{array}{l}\text { I. Feature } \\
\text { selection: } \\
\text { Cluster analysis } \\
\text { 2. Classification: } \\
\text { SVM }\end{array}$ & $\begin{array}{l}\text { To predict early } \\
\text { progression of } \\
\text { nonmetastatic NPC }\end{array}$ & $\begin{array}{l}\text { I. Model performance } \\
\text { - AUC: } 0.80 \text { [CI: } 0.73-0.89] \\
\text { - Sensitivity: } 0.92 \\
\text { - Specificity: } 0.52 \\
\text { - PPV: } 0.32 \\
\text { - NPV: } 0.96 \\
\text { 2. Model calibration } \\
\text { - Brier score: } 0.150\end{array}$ & $\begin{array}{l}\text { The proposed model trained with five clinical } \\
\text { features and radiomic features had the best } \\
\text { performance over the other models. Tumor shape } \\
\text { sphericity, first-order mean absolute deviation, } T \\
\text { stage, and overall stage were important factors } \\
\text { affecting 3-year disease progression. }\end{array}$ & $\begin{array}{l}\text { The use of radiomics can be used } \\
\text { for tumor diagnosis and risk } \\
\text { assessment. Shapley additive } \\
\text { explanations helped to find } \\
\text { relationship between features in the } \\
\text { model. }\end{array}$ & $\begin{array}{l}\text { I. The association between } \\
\text { Epstein-Barr virus and } \\
\text { progression-free survival was not } \\
\text { explored. } \\
\text { 2. Test-retest or time-dependent } \\
\text { variability of radiomic features } \\
\text { were not examined. } \\
\text { 3. The image resolutions of the } \\
\text { patient samples were diverse. }\end{array}$ \\
\hline $\begin{array}{l}\text { Jiao et al } \\
(2019)^{66} \\
\text { (China) }\end{array}$ & $\begin{array}{c}\text { NPC } \\
106 \\
\text { (IMRT plans) }\end{array}$ & $\begin{array}{l}\text { Machine } \\
\text { learning } \\
\text { (Miscellaneous } \\
\text { applications - } \\
\text { radiotherapy } \\
\text { planning) }\end{array}$ & $\begin{array}{l}\text { I. Prediction: } \\
\text { Distance-to-target } \\
\text { histogram (DTH) } \\
\text { general regression } \\
\text { neural network } \\
\text { (GRNN) and DTH } \\
\text { +Conformal plan } \\
\text { DVH GRNN }\end{array}$ & $\begin{array}{l}\text { To predict dose- } \\
\text { volume histograms of } \\
\text { OARs from IMRT plan }\end{array}$ & $\begin{array}{l}\text { I. DVH prediction accuracy (OARs) } \\
\text { - Dosimetric results } \\
\text { - Average R squared: } 0.95 \\
\text { - Average MAE: } 3.67\end{array}$ & $\begin{array}{l}\text { The addition of dosimetric information improved } \\
\text { the DVH prediction of the developed model. }\end{array}$ & $\begin{array}{l}\text { The study showed the prediction } \\
\text { capability of the model when patient } \\
\text { dosimetric information was added } \\
\text { to geometric information. }\end{array}$ & N/A \\
\hline
\end{tabular}




\begin{tabular}{|c|c|c|c|c|c|c|c|c|}
\hline $\begin{array}{l}\text { Jing et al } \\
(2019)^{17} \\
\text { (China) }\end{array}$ & $\begin{array}{l}\text { NPC and other } \\
\text { types } \\
6449 \\
\text { (Clinicopathological } \\
\text { and plasma EBV } \\
\text { DNA data) }\end{array}$ & $\begin{array}{l}\text { Deep learning } \\
\text { (Prognosis) }\end{array}$ & $\begin{array}{l}\text { I. Prediction: } \\
\text { RankDeepSurv }\end{array}$ & $\begin{array}{l}\text { Compare } \\
\text { RankDeepSurv with } \\
\text { other survival models } \\
\text { and clinical experts in } \\
\text { the analysis and } \\
\text { prognosis of four public } \\
\text { medical datasets and } \\
\text { NPC }\end{array}$ & $\begin{array}{l}\text { I. Predictive performance of survival } \\
\text { analysis } \\
\text { - C-index: } 0.68 \mid[0.678-0.684]\end{array}$ & $\begin{array}{l}\text { The RankDeepSurv had a better performance than } \\
\text { the other three referenced methods in four public } \\
\text { medical clinical datasets and in the NPC dataset } \\
\text { versus clinical experts }\end{array}$ & $\begin{array}{l}\text { The proposed model can assist } \\
\text { clinicians in providing more accurate } \\
\text { predictions for NPC recurrence }\end{array}$ & $N / A$ \\
\hline $\begin{array}{l}\text { Li et al }(2019)^{53} \\
\text { (China) }\end{array}$ & $\begin{array}{c}\text { NPC } \\
502 \\
\text { (CT images) }\end{array}$ & $\begin{array}{l}\text { Deep learning } \\
\text { (Auto- } \\
\text { contouring) }\end{array}$ & $\begin{array}{l}\text { I. Segmentation: } \\
\text { U-net }\end{array}$ & $\begin{array}{l}\text { To assess the } \\
\text { developed model's } \\
\text { accuracy in delineating } \\
\text { CT images }\end{array}$ & $\begin{array}{l}\text { I. Model performance } \\
\text { - DSC: } 0.74 \\
\text { - HD: } 12.85 \mathrm{~mm}\end{array}$ & $\begin{array}{l}\text { The modified U-net had a higher consistency and } \\
\text { performance than manual contouring, while using } \\
\text { less time per patient. }\end{array}$ & $\begin{array}{l}\text { The developed model has the } \\
\text { potential to help lighten clinicians' } \\
\text { workload and improve NPC } \\
\text { treatment outcomes. }\end{array}$ & $\mathrm{N} / \mathrm{A}$ \\
\hline $\begin{array}{l}\text { Liang et al } \\
(2019)^{52} \\
\text { (China) }\end{array}$ & $\begin{array}{c}\text { NPC } \\
185 \\
\text { (CT images) }\end{array}$ & $\begin{array}{l}\text { Deep learning } \\
\quad \text { (Auto- } \\
\text { contouring/ } \\
\text { Diagnosis) }\end{array}$ & $\begin{array}{l}\text { I. Recognition and } \\
\text { classification: } \\
\text { OAR detection and } \\
\text { segmentation } \\
\text { network (ODS net) }\end{array}$ & $\begin{array}{l}\text { To assess the } \\
\text { performance of the } \\
\text { model in detecting and } \\
\text { segmenting OARs }\end{array}$ & $\begin{array}{l}\text { I. Detection performance } \\
\text { - Sensitivity: I.000 [Cl: } 0.994-1.000] \\
\text { - Specificity: } 0.999[\text { Cl: } 0.997-1.000] \\
\text { 2. Segmentation performance } \\
\text { - DSC: } 0.934 \pm 0.04\end{array}$ & $\begin{array}{l}\text { The ODS net had good result in both detection and } \\
\text { segmentation performances. }\end{array}$ & $\begin{array}{l}\text { The fully automatic model may help } \\
\text { to facilitate therapy planning. }\end{array}$ & $\begin{array}{l}\text { I. The manual segmentation of } \\
\text { images by a radiologist may not be } \\
\text { consistent and may not be the } \\
\text { true standard of reference. } \\
\text { 2. The method was only examined } \\
\text { by one type of CT scan. } \\
\text { Investigation with other CT } \\
\text { scanners was needed. } \\
\text { 3. Contrast CT and MRI images } \\
\text { were not applied in the model, } \\
\text { affecting its performance. }\end{array}$ \\
\hline $\begin{array}{l}\text { Lin et al } \\
(2019)^{51} \\
\text { (China, Hong } \\
\text { Kong SAR, } \\
\text { China, and } \\
\text { Singapore) }\end{array}$ & $\begin{array}{c}\text { NPC } \\
\text { I02I } \\
\text { (MRI images) }\end{array}$ & $\begin{array}{l}\text { Deep learning } \\
\text { (Auto- } \\
\text { contouring) }\end{array}$ & $\begin{array}{l}\text { I. Feature } \\
\text { extraction: } \\
3 \mathrm{D} \text { CNN }\end{array}$ & $\begin{array}{l}\text { To evaluate the } \\
\text { developed model in } \\
\text { auto-contouring of } \\
\text { primary gross target } \\
\text { volume }\end{array}$ & $\begin{array}{l}\text { I. Model performance } \\
\text { - DSC: } 0.79[\text { [Cl: } 0.78-0.79] \\
\text { - ASSD: } 2.0 \mathrm{~mm}[\mathrm{Cl}: 1.9-2.1]\end{array}$ & $\begin{array}{l}\text { The model yielded good accuracy. It also helped } \\
\text { improve the contouring accuracy and time of } \\
\text { practitioners in the study. }\end{array}$ & $\begin{array}{l}\text { The model has the potential to help } \\
\text { tumor control and patient survival } \\
\text { by enhancing the delineation } \\
\text { accuracy and lower contouring } \\
\text { variation by different practitioners } \\
\text { and the time required. }\end{array}$ & $\begin{array}{l}\text { I. Low statistical power due to } \\
\text { small number of events. } \\
\text { 2. There may be memory bias } \\
\text { during evaluation. } \\
\text { 3. Poor contouring of } \\
\text { oropharynx, hypopharynx and } \\
\text { intracranial regions caused lower } \\
\text { accuracy at the cranial-caudal } \\
\text { edges. }\end{array}$ \\
\hline $\begin{array}{l}\text { Liu et al } \\
(2019)^{65} \\
\text { (China) }\end{array}$ & $\begin{array}{c}\text { NPC } \\
190 \\
\text { (Helical } \\
\text { tomotherapy plans) }\end{array}$ & $\begin{array}{l}\text { Deep learning } \\
\text { (Miscellaneous } \\
\text { applications - } \\
\text { radiotherapy } \\
\text { planning) }\end{array}$ & $\begin{array}{l}\text { I. Prediction: } \\
\text { U-ResNet-D }\end{array}$ & $\begin{array}{l}\text { To predict the three- } \\
\text { dimensional dose } \\
\text { distribution of helical } \\
\text { tomotherapy }\end{array}$ & $\begin{array}{l}\text { 1. Predictive performance } \\
\text { a) Dose difference } \\
\text { - Average: } 0.0 \% \pm 2.4 \% \\
\text { - Average MAE: } 1.9 \% \pm 1.8 \% \\
\text { b) Dosimetric index (DI) comparison } \\
\text { - Prediction accuracy (Dmean): } 2.4 \% \\
\text { - Prediction accuracy (Dmax): } 4.2 \% \\
\text { c) DSC: } 0.95-1\end{array}$ & $\begin{array}{l}\text { The U-ResNet-D model yielded good results in } \\
\text { predicting 3D dose distribution. }\end{array}$ & $\begin{array}{l}\text { The developed method has the } \\
\text { potential to increase the quality and } \\
\text { consistency of treatment plans. }\end{array}$ & $\begin{array}{l}\text { 1. Can only predict one type of } \\
\text { dose distribution. } \\
\text { 2. The model was unable to apply } \\
\text { the predicted 3D dose } \\
\text { distribution into a clinical plan. }\end{array}$ \\
\hline $\begin{array}{l}\text { Ma et al } \\
(2019)^{18} \text { (China } \\
\text { and USA) }\end{array}$ & $\begin{array}{l}\text { NPC } \\
90 \\
\text { (CT and MRI } \\
\text { images) }\end{array}$ & $\begin{array}{l}\text { Deep learning } \\
\text { (Auto- } \\
\text { contouring) }\end{array}$ & $\begin{array}{l}\text { I. Segmentation: } \\
\text { Multi-modality } \\
\text { CNN \& Combined } \\
\text { CNN }\end{array}$ & $\begin{array}{l}\text { To use MRI and CT in } \\
\text { NPC segmentation } \\
\text { with the proposed } \\
\text { models }\end{array}$ & $\begin{array}{l}\text { 1. Segmentation performance } \\
\text { - Sensitivity: } 0.718 \pm 0.121 \\
\text { - PPV: } 0.797 \pm 0.109 \\
\text { - DSC: } 0.752 \pm 0.043 \\
\text { - ASSD: } 1.062 \mathrm{~mm} \pm 0.298 \mathrm{~mm}\end{array}$ & $\begin{array}{l}\text { The proposed model out-performed the same } \\
\text { model but without multi-modal information fusion } \\
\text { and other existing CNN-based multi-modality } \\
\text { segmentation models. }\end{array}$ & $\begin{array}{l}\text { The proposed model was the first } \\
\text { CNN-based method to solve the } \\
\text { challenge of performing multi- } \\
\text { modality tumor contouring on } \\
\text { NPCs. }\end{array}$ & $N / A$ \\
\hline
\end{tabular}

(Continued) 
Table I (Continued).

\begin{tabular}{|c|c|c|c|c|c|c|c|c|}
\hline $\begin{array}{l}\text { Authors, } \\
\text { Year and } \\
\text { Country }\end{array}$ & $\begin{array}{l}\text { Site, No. of Cases } \\
\text { (Data Type) }\end{array}$ & $\begin{array}{l}\text { Al Subfield } \\
\text { (Application) }\end{array}$ & $\begin{array}{l}\text { Artificial } \\
\text { Intelligence } \\
\text { Methods and its } \\
\text { Application }\end{array}$ & Study Aim & Performance Metric (s) & Results & Conclusion & Limitations \\
\hline $\begin{array}{l}\text { Peng et al } \\
(2019)^{19} \\
\text { (China) }\end{array}$ & $\begin{array}{c}\text { NPC } \\
707 \\
\text { (PET/CT images) }\end{array}$ & $\begin{array}{l}\text { Deep learning } \\
\text { (Prognosis) }\end{array}$ & $\begin{array}{l}\text { I. Feature } \\
\text { extraction: } \\
\text { Nomogram using } \\
\text { Deep CNN }\end{array}$ & $\begin{array}{l}\text { To assess risk and guide } \\
\text { induction } \\
\text { chemotherapy for } \\
\text { patients }\end{array}$ & $\begin{array}{l}\text { 1. Prognostic performance } \\
\text { - C-index: } 0.722 \text { [Cl: } 0.652-0.792] \\
\text { - AUC ( } 5 \text { years): } 0.735\end{array}$ & $\begin{array}{l}\text { The DL-based radiomics nomogram out-performed } \\
\text { the EBV DNA-based model in risk stratification and } \\
\text { induction chemotherapy guiding }\end{array}$ & $\begin{array}{l}\text { The DL-based radiomics nomogram } \\
\text { can be used for individualized } \\
\text { treatment strategies. }\end{array}$ & $\begin{array}{l}\text { I. The follow-up period was too } \\
\text { short } \\
\text { 2. Data sample were only from } \\
\text { one center. } \\
\text { 3. There may be patient selection } \\
\text { biases confounded with radiomics } \\
\text { signatures and outcomes may be } \\
\text { present as induction } \\
\text { chemotherapy was not randomly } \\
\text { assigned to patients. }\end{array}$ \\
\hline $\begin{array}{l}\text { Rehioui et al } \\
(2019)^{20} \\
\text { (Morocco) }\end{array}$ & $\begin{array}{c}\text { NPC } \\
90 \\
\text { (Risk factors) }\end{array}$ & $\begin{array}{l}\text { Machine } \\
\text { learning } \\
\text { (Miscellaneous } \\
\text { applications - } \\
\text { Risk factor } \\
\text { identification) }\end{array}$ & $\begin{array}{l}\text { I. Prediction: } \\
\text { Clustering } \\
\text { algorithms (K } \\
\text { means, Expectation } \\
\text { Maximization } \\
\text { algorithm, density- } \\
\text { based algorithms } \\
\text { (DENCLUE and its } \\
\text { variants) }\end{array}$ & $\begin{array}{l}\text { Compare the prognosis } \\
\text { performance of } \\
\text { different algorithms }\end{array}$ & $\begin{array}{l}\text { I. Model performance } \\
\text { - Dunn Index: } 0.614 \\
\text { - Davies-Bouldin Index: } 2.110 \\
\text { - Compactness Index: } 0.781 \\
\text { - Accuracy: } 0.592 \\
\text { - Normalized Mutual Information: } \\
0.245 \\
\text { - Entropy: } 0.806\end{array}$ & $\begin{array}{l}\text { The density-based algorithms (DENCLUE and its } \\
\text { variants) obtained a better result than partitioning } \\
\text { or statistical models }\end{array}$ & $\begin{array}{l}\text { Familial history of cancer, living } \\
\text { conditions and tobacco } \\
\text { consumption are all associated with } \\
\text { advanced stage of NPC. }\end{array}$ & N/A \\
\hline $\begin{array}{l}\text { Zhong et al } \\
(2019)^{50} \\
\text { (China) }\end{array}$ & $\begin{array}{c}\text { NPC } \\
140 \\
\text { (CT images) }\end{array}$ & $\begin{array}{l}\text { Deep learning } \\
\text { (Auto- } \\
\text { contouring) }\end{array}$ & $\begin{array}{l}\text { I. Segmentation: } \\
\text { Boosting Resnet \& } \\
\text { Voxelwise Resnet }\end{array}$ & $\begin{array}{l}\text { To assess the proposed } \\
\text { model in delineating } \\
\text { OARs }\end{array}$ & $\begin{array}{l}\text { I. Segmentation performance } \\
\text { - DSC: } 0.9188 \pm 0.0351 \\
-95 \mathrm{HD}: 3.15 \mathrm{~mm} \pm 0.58 \mathrm{~mm} \\
\text { - Volume overlap error: } 14.83 \% \\
\pm 5.26 \%\end{array}$ & $\begin{array}{l}\text { The proposed cascaded method gave a significantly } \\
\text { better performance than other existing single } \\
\text { network architecture or segmentation algorithms. }\end{array}$ & $\begin{array}{l}\text { The study showed the effectiveness } \\
\text { of the developed model when auto- } \\
\text { contouring OARs and the benefits } \\
\text { of using the cascaded DL structure. }\end{array}$ & N/A \\
\hline $\begin{array}{l}\text { Zou et al } \\
(2019)^{21} \text { (China } \\
\text { and United } \\
\text { Kingdom) }\end{array}$ & $\begin{array}{c}\text { NPC } \\
99 \\
\text { (CT and MRI } \\
\text { images) }\end{array}$ & $\begin{array}{l}\text { Deep learning } \\
\text { (Miscellaneous } \\
\text { applications - } \\
\quad \text { Image } \\
\text { registration) }\end{array}$ & $\begin{array}{l}\text { I. Image } \\
\text { registration: } \\
\text { Full convolution } \\
\text { network, CNN \& } \\
\text { Random sample } \\
\text { consensus }\end{array}$ & $\begin{array}{l}\text { To develop a model for } \\
\text { image registration }\end{array}$ & $\begin{array}{l}\text { I. Image registration } \\
\text { - Precision' } \\
\text { - Recall' } \\
\text { - Target registration error }(\text { TRE })^{\mathrm{a}}\end{array}$ & $\begin{array}{l}\text { The proposed method with additional use of } \\
\text { transfer learning and fine-tuning out-performed } \\
\text { both the proposed method and scale invariant } \\
\text { feature transform (SIFT). }\end{array}$ & $\begin{array}{l}\text { The use of transfer learning and fine } \\
\text { tuning in the proposed model is } \\
\text { promising in improving image } \\
\text { registration. }\end{array}$ & N/A \\
\hline $\begin{array}{l}\text { Abd Ghani et al } \\
(2020)^{61} \\
\text { (Malaysia, Iraq } \\
\text { and India) }\end{array}$ & $\begin{array}{c}\text { NPC } \\
381 \\
\text { (Endoscopic images) }\end{array}$ & $\begin{array}{l}\text { Machine } \\
\text { learning } \\
\text { (Diagnosis) }\end{array}$ & $\begin{array}{l}\text { I. Classification: } \\
\text { KNN, Linear SVM } \\
\text { \& ANN }\end{array}$ & $\begin{array}{l}\text { To develop a model } \\
\text { with endoscopic } \\
\text { images for NPC } \\
\text { identification }\end{array}$ & $\begin{array}{l}\text { I. Classification performance } \\
\text { - Sensitivity: } 0.925 \\
\text { - Specificity: } 0.937 \\
\text { - Accuracy: } 0.947\end{array}$ & $\begin{array}{l}\text { The majority rule for decision-based fusion } \\
\text { technique had a significantly lower performance } \\
\text { than using a single best performing feature scheme } \\
\text { for the SVM classifier, which uses pair-wise fusion of } \\
\text { only two features. }\end{array}$ & $\begin{array}{l}\text { A fully automated NPC detection } \\
\text { model with good accuracy was } \\
\text { developed. Although the proposed } \\
\text { method had a high accuracy, the } \\
\text { single best performing feature } \\
\text { scheme for the SVM classifier } \\
\text { outperforms it. }\end{array}$ & N/A \\
\hline
\end{tabular}




\begin{tabular}{|c|c|c|c|c|c|c|c|c|}
\hline $\begin{array}{l}\text { Bai et al } \\
(2020)^{64} \text { (China } \\
\text { and USA) }\end{array}$ & $\begin{array}{c}\text { NPC } \\
140 \\
\text { (IMRT plans) }\end{array}$ & $\begin{array}{l}\text { Machine } \\
\text { learning } \\
\text { (Miscellaneous } \\
\text { applications - } \\
\text { radiotherapy } \\
\text { planning) }\end{array}$ & $\begin{array}{l}\text { I. Prediction: } \\
\text { ANN }\end{array}$ & $\begin{array}{l}\text { To explore viability of a } \\
\text { model for knowledge- } \\
\text { based automated } \\
\text { intensity-modulated } \\
\text { radiation therapy } \\
\text { planning }\end{array}$ & $\begin{array}{l}\text { 1. Plan quality } \\
\text { - } \mathrm{DVH}^{\mathrm{a}} \\
\text { - Dose distribution } \\
\text { - Monitor unit: } 685.04 \pm 59.63 \\
\text { - Planning duration: } 9.85 \mathrm{~min} \pm 1.13 \mathrm{~min}\end{array}$ & $\begin{array}{l}\text { The proposed model had a similar performance but } \\
\text { with a higher efficiency in treatment planning when } \\
\text { compared with manual planning. }\end{array}$ & $\begin{array}{l}\text { The proposed technique can } \\
\text { significantly reduce the treatment } \\
\text { planning time while maintaining the } \\
\text { same plan quality. }\end{array}$ & $\mathrm{N} / \mathrm{A}$ \\
\hline $\begin{array}{l}\text { Chen et al } \\
(2020)^{22} \\
\text { (China) }\end{array}$ & $\begin{array}{c}\text { NPC } \\
\text { 149 } \\
\text { (MRI images) }\end{array}$ & $\begin{array}{l}\text { Deep learning } \\
\text { (Auto- } \\
\text { contouring) }\end{array}$ & $\begin{array}{l}\text { I. Segmentation: } \\
\text { Multi-modality MRI } \\
\text { fusion network }\end{array}$ & $\begin{array}{l}\text { To evaluate the } \\
\text { segmentation } \\
\text { performance of a } \\
\text { model which uses TI, } \\
\text { T2 and contrast- } \\
\text { enhanced TI MRI }\end{array}$ & $\begin{array}{l}\text { I. Segmentation performance } \\
\text { - DSC: } 0.7238 \pm 0.1099 \\
\text { - HD: } 18.31 \mathrm{~mm} \pm 16.73 \mathrm{~mm} \\
\text { - ASSD: } 2.07 \mathrm{~mm} \pm 2.32 \mathrm{~mm}\end{array}$ & $\begin{array}{l}\text { In comparison with other existing DL algorithms, } \\
\text { the proposed model had the best segmentation } \\
\text { performance. }\end{array}$ & $\begin{array}{l}\text { Multi-modality MRI is useful to the } \\
\text { proposed model for NPC } \\
\text { delineation. }\end{array}$ & $\mathrm{N} / \mathrm{A}$ \\
\hline $\begin{array}{l}\text { Chen et al } \\
(2020)^{68} \\
\text { (China) }\end{array}$ & $\begin{array}{c}\text { NPC } \\
99 \\
\text { (VMAT plans) }\end{array}$ & $\begin{array}{l}\text { Machine } \\
\text { learning } \\
\text { (Miscellaneous } \\
\text { applications - } \\
\text { radiotherapy } \\
\text { planning) }\end{array}$ & $\begin{array}{l}\text { I. Prediction: } \\
\text { ANN }\end{array}$ & $\begin{array}{l}\text { To develop models for } \\
\text { radiotherapy planning } \\
\text { for planning quality } \\
\text { control }\end{array}$ & $\begin{array}{l}\text { I. Plan quality } \\
\text { a) } \mathrm{DVH}^{\mathrm{a}} \\
\text { b) Dose distribution }\end{array}$ & $\begin{array}{l}\text { The developed ANN model had a lower capability } \\
\text { than the junior physicist in designing radiotherapy } \\
\text { plans. }\end{array}$ & $\begin{array}{l}\text { The proposed model enhancing the } \\
\text { quality and stability of individualized } \\
\text { radiotherapy planning. }\end{array}$ & $N / A$ \\
\hline $\begin{array}{l}\text { Chuang et al } \\
(2020)^{23} \\
\text { (Taiwan) }\end{array}$ & $\begin{array}{l}\text { NPC } \\
726 \\
(\text { WSIs) }\end{array}$ & $\begin{array}{l}\text { Deep learning } \\
\text { (Diagnosis) }\end{array}$ & $\begin{array}{l}\text { I. Classification: } \\
\text { Patch-level deep } \\
\text { CNN \& Slide-level } \\
\text { deep CNN }\end{array}$ & $\begin{array}{l}\text { To assess proposed } \\
\text { model in detecting } \\
\text { NPC in biopsies }\end{array}$ & $\begin{array}{l}\text { I. Diagnostic performance } \\
\text { - AUC: } 0.9900 \pm 0.004 \\
\text { - Sensitivity } \\
\text { - Specificity }\end{array}$ & $\begin{array}{l}\text { The slide-level model had a better performance } \\
\text { than pathology residents. However, its diagnostic } \\
\text { ability is slightly worse than both attending } \\
\text { pathologists and the chief resident. }\end{array}$ & $\begin{array}{l}\text { The study demonstrated for the } \\
\text { first time that DL algorithms can } \\
\text { identify NPC in biopsies. }\end{array}$ & $N / A$ \\
\hline $\begin{array}{l}\text { Cui et al } \\
(2020)^{39} \\
\text { (China) }\end{array}$ & $\begin{array}{c}\text { NPC } \\
792 \\
\text { (MRI images) }\end{array}$ & $\begin{array}{l}\text { Machine } \\
\text { learning and } \\
\text { deep learning } \\
\text { (Prognosis) }\end{array}$ & $\begin{array}{l}\text { I. Feature } \\
\text { selection: } \\
\text { Generalized linear } \\
\text { model (ridge/lasso), } \\
\text { XRT, Gradient } \\
\text { boosting machine, } \\
\text { RF \& DL } \\
\text { (Unknown) }\end{array}$ & $\begin{array}{l}\text { To assign prediction } \\
\text { scores to NPC patients } \\
\text { and compare with the } \\
\text { current clinical staging } \\
\text { system }\end{array}$ & $\begin{array}{l}\text { I. Prognostic performance } \\
\text { a) AUC } \\
\text { - OS: } 0.796 \text { (s.d. }=0.044) \\
\text { - DMFS: } 0.752 \text { (s.d. }=0.042) \\
\text { - LRFS: } 0.72 \text { (s.d. }=0.052) \\
\text { b) Specificity } \\
\text { - OS: } 0.72 \text { (s.d. }=0.061) \\
\text { - DMFS: } 0.576 \text { (s.d }=0.114) \\
\text { - LRFS: } 0.540 \text { (s.d. }=0.153 \text { ) } \\
\text { c) Test error } \\
\text { - OS: } 0.208 \text { (s.d. }=0.037 \text { ) } \\
\text { - DMFS: } 0.271 \text { (s.d. }=0.052) \\
\text { - LRFS: } 0.287 \text { (s.d. }=0.050)\end{array}$ & $\begin{array}{l}\text { The new scoring system had a better prognostic } \\
\text { performance than the TNM/AJCC system in } \\
\text { predicting treatment outcome for NPC }\end{array}$ & $\begin{array}{l}\text { The new scoring system has the } \\
\text { potential to improve image data- } \\
\text { based clinical predictions and } \\
\text { precision oncology }\end{array}$ & $\begin{array}{l}\text { 1. The time to event was not } \\
\text { considered. } \\
\text { 2. No testing set. } \\
\text { 3. Only data from MRI images } \\
\text { were used for the scoring system }\end{array}$ \\
\hline $\begin{array}{l}\text { Diao et al } \\
(2020)^{60} \\
\text { (China) }\end{array}$ & $\begin{array}{l}\text { NPC } \\
1970 \\
\text { (WSIs) }\end{array}$ & $\begin{array}{l}\text { Deep learning } \\
\text { (Diagnosis) }\end{array}$ & $\begin{array}{l}\text { I. Classification: } \\
\text { Inception-v3 }\end{array}$ & $\begin{array}{l}\text { To assess the } \\
\text { pathologic diagnosis of } \\
\text { NPC with the } \\
\text { proposed model }\end{array}$ & $\begin{array}{l}\text { I. Diagnostic performance } \\
\text { - AUC: } 0.930 \\
\text { - Sensitivity: } 0.929 \\
\text { - Specificity: } 0.801 \\
\text { - Accuracy: } 0.905 \\
\text { - Jaccard index: } 0.879 \\
\text { - Euclidean distance: } 0.242 \\
\text { - Kappa factor: } 0.842\end{array}$ & $\begin{array}{l}\text { Inception-v3 performed better than the junior and } \\
\text { intermediate pathologists, but was worse than the } \\
\text { senior pathologist in accuracy, specificity, sensitivity, } \\
\text { AUC and consistency. }\end{array}$ & $\begin{array}{l}\text { The proposed model can be used to } \\
\text { support pathologists in clinical } \\
\text { diagnosis by acting as a diagnostic } \\
\text { reference. }\end{array}$ & $\begin{array}{l}\text { 1. Improvement in the model's } \\
\text { design is required. } \\
\text { 2. The model only identifies if the } \\
\text { tumour was cancerous but not its } \\
\text { subtype. } \\
\text { 3. Limited sample size. }\end{array}$ \\
\hline
\end{tabular}




\begin{tabular}{|c|c|c|c|c|c|c|c|c|}
\hline $\begin{array}{l}\text { Authors, } \\
\text { Year and } \\
\text { Country }\end{array}$ & $\begin{array}{l}\text { Site, No. of Cases } \\
\text { (Data Type) }\end{array}$ & $\begin{array}{l}\text { Al Subfield } \\
\text { (Application) }\end{array}$ & $\begin{array}{l}\text { Artificial } \\
\text { Intelligence } \\
\text { Methods and its } \\
\text { Application }\end{array}$ & Study Aim & Performance Metric (s) & Results & Conclusion & Limitations \\
\hline $\begin{array}{l}\text { Du et al } \\
(2020)^{59} \\
\text { (China, USA } \\
\text { and Canada) }\end{array}$ & $\begin{array}{c}\text { NPC } \\
76 \\
\text { (PET/CT images) }\end{array}$ & $\begin{array}{l}\text { Machine } \\
\text { learning } \\
\text { (Diagnosis) }\end{array}$ & $\begin{array}{l}\text { I. Classifications: } \\
\text { Decision tree, } \\
\text { KNN, Linear } \\
\text { discriminant } \\
\text { analysis, LR, Naiive } \\
\text { bayes, RF \& SVM } \\
\text { with radial basis } \\
\text { function kernel } \\
\text { SVM }\end{array}$ & $\begin{array}{l}\text { To evaluate and } \\
\text { compare different } \\
\text { machine learning } \\
\text { methods in } \\
\text { differentiating local } \\
\text { recurrence and } \\
\text { inflammation }\end{array}$ & $\begin{array}{l}\text { I. Diagnostic performance } \\
\text { - AUC: } 0.883 \text { [Cl: } 0.675-0.979] \\
\text { - Sensitivity: } 0.833 \\
\text { - Specificity: } 1.000 \\
\text { - Reliability (test error): } 0.091 \text { [Cl: } \\
0.001-0.244]\end{array}$ & $\begin{array}{l}\text { The combination of fisher score with KNN, FSCR } \\
\text { with support vector machines with RBF-SVM, fisher } \\
\text { score with RF, and minimum redundancy maximum } \\
\text { relevance with RBF-SVM had significantly better } \\
\text { performance in accuracy, sensitivity, specificity and } \\
\text { reliability than other combination of techniques. }\end{array}$ & $\begin{array}{l}\text { Several methods to integrate ML } \\
\text { algorithms with radiomics have the } \\
\text { potential to improve NPC } \\
\text { diagnostics. }\end{array}$ & $\begin{array}{l}\text { 1. Limited by the retrospective } \\
\text { nature and small sample size from } \\
\text { one source. } \\
\text { 2. Only common feature selection } \\
\text { and classification techniques were } \\
\text { chosen. No parameter tuning was } \\
\text { performed. } \\
\text { 3. Clinical parameters and } \\
\text { genomic data were not included. }\end{array}$ \\
\hline $\begin{array}{l}\text { Guo et al } \\
(2020)^{24} \\
\text { (China) }\end{array}$ & $\begin{array}{c}\text { NPC } \\
120 \\
\text { (3D MRI images) }\end{array}$ & $\begin{array}{l}\text { Deep learning } \\
\quad \text { (Auto- } \\
\text { contouring) }\end{array}$ & $\begin{array}{l}\text { 1. Segmentation: } \\
\text { 3D CNN with } \\
\text { multi-scale pyramid } \\
\text { network }\end{array}$ & $\begin{array}{l}\text { To evaluate the } \\
\text { segmentation } \\
\text { performance of a } \\
\text { model }\end{array}$ & $\begin{array}{l}\text { I. Segmentation performance } \\
\text { - DSC: } 0.7370 \\
\text { - ASSD: } 1.214 \mathrm{~mm} \\
\text { - FI-score: } 0.7540\end{array}$ & $\begin{array}{l}\text { The developed model out-performed the other DL } \\
\text { models. Furthermore, the Jaccard loss function } \\
\text { improved the segmentation performance of all } \\
\text { models substantially. }\end{array}$ & $\begin{array}{l}\text { The Jaccard loss function solved the } \\
\text { issue of extreme foreground and } \\
\text { background imbalance in image } \\
\text { segmentation. However, further } \\
\text { validation is required. }\end{array}$ & N/A \\
\hline $\begin{array}{l}\text { Jing et al } \\
(2020)^{25} \\
\text { (China) }\end{array}$ & $\begin{array}{l}\text { NPC } \\
\text { I846 } \\
\text { (3D MRI images and } \\
\text { clinicopathological } \\
\text { data) }\end{array}$ & $\begin{array}{l}\text { Deep learning } \\
\text { (Prognosis) }\end{array}$ & $\begin{array}{l}\text { I. Prediction: } \\
\text { End-to-end multi- } \\
\text { modality deep } \\
\text { survival network } \\
\text { (MDSN) }\end{array}$ & $\begin{array}{l}\text { To predict and } \\
\text { categorize the risk } \\
\text { scores of NPC patients }\end{array}$ & $\begin{array}{l}\text { I. Model performance } \\
\text { - C-index: } 0.65 \text { I }\end{array}$ & $\begin{array}{l}\text { The end-to-end MDSN had a better performance } \\
\text { than the other four survival methods. } \\
\text { The integration of clinical stages into the MDSN } \\
\text { model further improves its performance. }\end{array}$ & $\begin{array}{l}\text { MDSN has the potential to support } \\
\text { clinicians in making treatment } \\
\text { decisions. }\end{array}$ & N/A \\
\hline $\begin{array}{l}\text { Ke et al } \\
(2020)^{49} \\
\text { (China) }\end{array}$ & $\begin{array}{c}\text { NPC } \\
4100 \\
\text { (3D MRI images) }\end{array}$ & $\begin{array}{l}\text { Deep learning } \\
\quad \text { (Auto- } \\
\text { contouring/ } \\
\text { Diagnosis) }\end{array}$ & $\begin{array}{l}\text { I. Classification and } \\
\text { segmentation: } \\
\text { Self-constrained 3D } \\
\text { DenseNet model }\end{array}$ & $\begin{array}{l}\text { To assess the detection } \\
\text { and segmentation } \\
\text { ability of the developed } \\
\text { model }\end{array}$ & $\begin{array}{l}\text { I. Diagnostic performance } \\
\text { - AUC: } 0.976 \text { [Cl: } 0.966-0.987] \\
\text { - Sensitivity: } 0.9968 \text { [Cl: } 0.9792- \\
0.9998] \\
\text { - Specificity: } 0.9167 \text { [Cl: } 0.8377- \\
0.9607] \\
\text { - Accuracy: } 0.9777 \text { [Cl: } 0.9566- \\
0.989 \text { ] } \\
\text { - PPV: } 0.9909 \text { [Cl: } 0.9861-0.994 \mid] \\
\text { - NPV: } 0.9637 \text { [Cl: } 0.9473-0.9753] \\
\text { 2. Segmentation performance } \\
\text { - DSC: } 0.77 \pm 0.07\end{array}$ & $\begin{array}{l}\text { The model had encouraging segmentation ability } \\
\text { and the diagnostic performance of the proposed } \\
\text { model obtained a better result than that of } \\
\text { experienced radiologists. }\end{array}$ & $\begin{array}{l}\text { The developed model may be able } \\
\text { to improve the diagnostic efficiency } \\
\text { and assist in clinical practice. }\end{array}$ & $\begin{array}{l}\text { 1. No external validation. } \\
\text { 2. The model only trained with } \\
\text { MRI images. Other clinical factors } \\
\text { were not considered. } \\
\text { 3. Patients diagnosed with benign } \\
\text { hyperplasia were not confirmed } \\
\text { with histopathology. }\end{array}$ \\
\hline $\begin{array}{l}\text { Liu et al } \\
(2020)^{38} \\
\text { (China) }\end{array}$ & $\begin{array}{l}\text { NPC } \\
1055 \\
\text { (WSIs) }\end{array}$ & $\begin{array}{l}\text { Deep learning } \\
\text { (Prognosis) }\end{array}$ & $\begin{array}{l}\text { I. Risk score } \\
\text { calculation: } \\
\text { DeepSurv }\end{array}$ & $\begin{array}{l}\text { To assess the survival } \\
\text { risk of NPC patients in } \\
\text { order to make } \\
\text { treatment decisions }\end{array}$ & $\begin{array}{l}\text { I. Survival risk assessment } \\
\text {-C-index: } 0.723\end{array}$ & $\begin{array}{l}\text { DeepSurv to analyze the pathological microscopic } \\
\text { features was a higher independent prognostic risk } \\
\text { factor than EBV DNA copies and } N \text { stage }\end{array}$ & $\begin{array}{l}\text { DeepSurv to analyze the } \\
\text { pathological microscopic features } \\
\text { can be used as a reliable tool for } \\
\text { assessing survival risk in NPC } \\
\text { patients. }\end{array}$ & $\begin{array}{l}\text { I. Decreased generalizability } \\
\text { when applied to other centers or } \\
\text { populations. } \\
\text { 2. Samples only consisted of } \\
\text { undifferentiated non- } \\
\text { keratinization NPC in the } \\
\text { endemic region. } \\
\text { 3. The principle in which } \\
\text { pathological microfeatures assist } \\
\text { in guiding treatment is unknown }\end{array}$ \\
\hline
\end{tabular}




\begin{tabular}{|c|c|c|c|c|c|c|c|c|}
\hline $\begin{array}{l}\text { Men et al } \\
(2020)^{47} \\
\text { (China) }\end{array}$ & $\begin{array}{c}\text { NPC } \\
600 \\
\text { (CT images) }\end{array}$ & $\begin{array}{l}\text { Deep learning } \\
\quad \text { (Auto- } \\
\text { contouring) }\end{array}$ & $\begin{array}{l}\text { I. Segmentation: } \\
\text { CNN } \\
\text { 2. Classification: } \\
\text { Resnet-10I }\end{array}$ & $\begin{array}{l}\text { To assess the proposed } \\
\text { method to improve } \\
\text { segmentation } \\
\text { constantly with less } \\
\text { labelling effort }\end{array}$ & $\begin{array}{l}\text { 1. Classification Performance } \\
\text { - AUC: } 0.91 \\
\text { - Sensitivity: } 0.92 \\
\text { - Specificity: } 0.90 \\
\text { - Accuracy: } 0.92 \\
\text { 2. Segmentation performance } \\
\text { - DSC: } 0.86 \pm 0.02\end{array}$ & $\begin{array}{l}\text { The proposed method could improve segmentation } \\
\text { performance, while reducing the amount of } \\
\text { labelling required. }\end{array}$ & $\begin{array}{l}\text { The developed model decreased the } \\
\text { amount of labelling and boosted } \\
\text { segmentation performance by } \\
\text { constantly obtaining, fine-tuning and } \\
\text { transferring knowledge over long } \\
\text { periods of time. }\end{array}$ & $\begin{array}{l}\text { I. The effect of the number of } \\
\text { locked layers were not } \\
\text { investigated. } \\
2 \text {. The study did not use } 3 \mathrm{D} \\
\text { segmentation model and counted } \\
\text { the labelling reduction based on } \\
\text { slices, resulting in poor automatic } \\
\text { segmentation in several slices for } \\
\text { nearly all patients }\end{array}$ \\
\hline $\begin{array}{l}\text { Mohammed } \\
\text { et al }(2020)^{26} \\
\text { (Malaysia, Iraq } \\
\text { and India) }\end{array}$ & $\begin{array}{c}\text { NPC } \\
381 \\
\text { (Endoscopic images) }\end{array}$ & $\begin{array}{l}\text { Machine } \\
\text { learning } \\
\text { (Diagnosis) }\end{array}$ & $\begin{array}{l}\text { I. Classification: } \\
\text { Multilayer } \\
\text { perceptron ANN }\end{array}$ & $\begin{array}{l}\text { To detect NPC from } \\
\text { endoscopic images }\end{array}$ & $\begin{array}{l}\text { I. Classification performance } \\
\text { - AUC: } 0.931 \pm 0.017 \\
\text { - Sensitivity: } 0.9543 \pm 0.0165 \\
\text { - Specificity: } 0.9578 \pm 0.0221 \\
\text { - Accuracy: } 0.9566 \pm 0.0175 \\
\text { - PPV: } 0.9455 \pm 0.0433\end{array}$ & $\begin{array}{l}\text { The developed models yielded good results and } \\
\text { ANN,50-50-A, had the best performance. }\end{array}$ & $\begin{array}{l}\text { The study was the first to } \\
\text { consolidate diverse features into } \\
\text { one fully automated classifier. }\end{array}$ & $\begin{array}{l}\text { 1. Insufficient sample size and } \\
\text { limited changeability. } \\
\text { 2. Possible misidentification of } \\
\text { endoscopy image data by experts. }\end{array}$ \\
\hline $\begin{array}{l}\text { Wang et al } \\
(2020)^{27} \\
\text { (China, USA } \\
\text { and Thailand) }\end{array}$ & $\begin{array}{c}\text { NPC } \\
186 \\
\text { (CT and MRI } \\
\text { images) }\end{array}$ & $\begin{array}{l}\text { Machine } \\
\text { learning } \\
\text { (Radiation- } \\
\text { induced injury } \\
\text { diagnosis) }\end{array}$ & $\begin{array}{l}\text { I. Prediction: } \\
\text { XGBoost }\end{array}$ & $\begin{array}{l}\text { To assess the feasibility } \\
\text { in developing a model } \\
\text { for predicting } \\
\text { radiation-related } \\
\text { fibrosis }\end{array}$ & $\begin{array}{l}\text { 1. Predictive performance } \\
\text { - AUC: } 0.69 \\
\text { - Sensitivity: } 0.0215 \\
\text { - Specificity: } 0.9866 \\
\text { - Accuracy: } 0.65\end{array}$ & $\begin{array}{l}\text { The proposed model trained with } \mathrm{CT} \text { images had a } \\
\text { better diagnostic accuracy than when using MRI } \\
\text { features. }\end{array}$ & $\begin{array}{l}\text { The proposed technique can be } \\
\text { used to perform patient specific } \\
\text { treatments by adjusting the } \\
\text { administered dose on the neck, } \\
\text { which can minimize the side effects. }\end{array}$ & $\begin{array}{l}\text { 1. There is subject bias in fibrosis } \\
\text { grading. } \\
\text { 2. The radiomic analysis protocol } \\
\text { is impractical to be used in daily } \\
\text { clinical practice. } \\
\text { 3. The investigation does not } \\
\text { differentiate between radiation- } \\
\text { induced fibrosis and residual or } \\
\text { recurrent tumour. }\end{array}$ \\
\hline $\begin{array}{l}\text { Wang et al } \\
(2020)^{48} \\
\text { (China) }\end{array}$ & $\begin{array}{c}\text { NPC } \\
205 \\
\text { (CT images) }\end{array}$ & $\begin{array}{l}\text { Deep learning } \\
\quad \text { (Auto- } \\
\text { contouring) }\end{array}$ & $\begin{array}{l}\text { I. Feature } \\
\text { extraction: } \\
\text { Modified 3D U-Net } \\
\text { based on Res-block } \\
\text { and SE-block }\end{array}$ & $\begin{array}{l}\text { To develop a model for } \\
\text { automatic delineation } \\
\text { of NPC in computed } \\
\text { tomography }\end{array}$ & $\begin{array}{l}\text { I. Delineation accuracy } \\
\text { - Precision: } 0.7538 \\
\text { - Sensitivity: } 0.7634 \\
\text { - DSC: } 0.7372 \\
\text { - HD } 95: 4.96 \mathrm{~mm} \\
\text { - ASSD: } 1.47 \mathrm{~mm}\end{array}$ & $\begin{array}{l}\text { The proposed model out-performed the other } \\
\text { methods in the experiment. In addition, using CT } \\
\text { combined with contrast-enhanced-CT instead of } \\
\mathrm{CT} \text { alone improves the performance of all models. }\end{array}$ & $\begin{array}{l}\text { The study showed that the } \\
\text { proposed fully automated model has } \\
\text { promise in helping clinicians in } 3 \mathrm{D} \\
\text { delineation of tumour during } \\
\text { radiotherapy planning by minimize } \\
\text { delineation variability. }\end{array}$ & $\begin{array}{l}\text { I. The patient samples were all } \\
\text { from one medical center. } \\
\text { 2. The model could only } \\
\text { automatically delineate the } \\
\text { nasopharynx gross tumour } \\
\text { volume }\end{array}$ \\
\hline $\begin{array}{l}\text { Xie et al } \\
(2020)^{37} \text { (Hong } \\
\text { Kong SAR, } \\
\text { China) }\end{array}$ & $\begin{array}{c}\text { NPC } \\
166 \\
\text { (PET/CT images) }\end{array}$ & $\begin{array}{l}\text { Machine } \\
\text { learning } \\
\text { (Prognosis) }\end{array}$ & $\begin{array}{l}\text { I. Prediction: } \\
\text { LR, SVM, RF \& } \\
\text { XGBoost }\end{array}$ & $\begin{array}{l}\text { To investigate the effect } \\
\text { of re-sampling } \\
\text { technique and machine } \\
\text { learning classifiers on } \\
\text { radiomics-based model }\end{array}$ & $\begin{array}{l}\text { I. Predictive performance } \\
\text { - AUC: } 0.66 \\
\text { - Geometric mean score: } 0.65 \\
\text { - Precision: } 0.90 \\
\text { - Recall: } 0.74 \\
\text { - F-measures: } 0.81\end{array}$ & $\begin{array}{l}\text { The combination of adaptive synthetic re-sampling } \\
\text { technique and SVM classifier gave the best } \\
\text { performance }\end{array}$ & $\begin{array}{l}\text { Re-sampling technique significantly } \\
\text { improved the prediction } \\
\text { performance of imbalanced datasets }\end{array}$ & $\begin{array}{l}\text { 1. The relatively small number of } \\
\text { instances and features in the } \\
\text { retrospective dataset may reduce } \\
\text { the generalizability to other kinds } \\
\text { of cancer. } \\
\text { 2. The paper mainly focused on } \\
\text { the data level approach using re- } \\
\text { sampling technique }\end{array}$ \\
\hline $\begin{array}{l}\text { Xue et al } \\
(2020)^{69} \\
\text { (China) }\end{array}$ & $\begin{array}{c}\text { NPC } \\
150 \\
\text { (Combined CT and } \\
\text { MRI images) }\end{array}$ & $\begin{array}{l}\text { Deep learning } \\
\quad \text { (Auto- } \\
\text { contouring) }\end{array}$ & $\begin{array}{l}\text { I. Segmentation: } \\
\text { Deeplabv3+ } \\
\text { 2. Feature } \\
\text { extraction: } \\
\text { MobileNetV2 }\end{array}$ & $\begin{array}{l}\text { To evaluate the } \\
\text { performance of the } \\
\text { proposed model in } \\
\text { segmenting high risk } \\
\text { tumors }\end{array}$ & $\begin{array}{l}\text { I. Segmentation accuracy } \\
\text { - DSC: } 0.76 \pm 0.11 \\
\text { - HD: } 10.9 \mathrm{~mm} \pm 8.6 \mathrm{~mm} \\
\text { - ASSD: } 3.4 \mathrm{~mm} \pm 2.0 \mathrm{~mm} \\
\text { - Jaccard index: } 0.63 \pm 0.13\end{array}$ & $\begin{array}{l}\text { The developed model had a better performance } \\
\text { when compared with the U-net model. Its results } \\
\text { were closer to manual contouring. }\end{array}$ & $\begin{array}{l}\text { The developed model has promise } \\
\text { in increasing the effectiveness and } \\
\text { consistency of primary tumour } \\
\text { gross target volume delineation for } \\
\text { NPC patients. }\end{array}$ & $\begin{array}{l}\text { 1. Insufficient training data. } \\
\text { 2. Delineation variability between } \\
\text { clinicians. } \\
\text { 3. MRI images were not used for } \\
\text { training the model. }\end{array}$ \\
\hline
\end{tabular}


Table I (Continued).

\begin{tabular}{|c|c|c|c|c|c|c|c|c|}
\hline $\begin{array}{l}\text { Authors, } \\
\text { Year and } \\
\text { Country }\end{array}$ & $\begin{array}{l}\text { Site, No. of Cases } \\
\text { (Data Type) }\end{array}$ & $\begin{array}{l}\text { Al Subfield } \\
\text { (Application) }\end{array}$ & $\begin{array}{l}\text { Artificial } \\
\text { Intelligence } \\
\text { Methods and its } \\
\text { Application }\end{array}$ & Study Aim & Performance Metric (s) & Results & Conclusion & Limitations \\
\hline $\begin{array}{l}\text { Xue et al } \\
(2020)^{46} \\
\text { (China) }\end{array}$ & $\begin{array}{c}\text { NPC } \\
150 \\
\text { (Combined CT and } \\
\text { MRI images) }\end{array}$ & $\begin{array}{l}\text { Deep learning } \\
\quad \text { (Auto- } \\
\text { contouring) }\end{array}$ & $\begin{array}{l}\text { I. Segmentation: } \\
\text { Sl-net }\end{array}$ & $\begin{array}{l}\text { To assess the model's } \\
\text { ability to segment high- } \\
\text { risk tumors }\end{array}$ & $\begin{array}{l}\text { I. Segmentation performance } \\
\text { - DSC: } 0.84 \pm 0.04 \\
\text { - HD: } 8.7 \mathrm{~mm} \pm 2.5 \mathrm{~mm} \\
\text { - ASSD: } 2.8 \mathrm{~mm} \pm 1.0 \mathrm{~mm} \\
\text { - Jaccard index: } 0.74 \pm 0.05\end{array}$ & $\begin{array}{l}\text { The SI-Net model had a better segmentation } \\
\text { performance than the U-net model. The mean } \\
\text { contouring time of the model is also less than when } \\
\text { performed manually. }\end{array}$ & $\begin{array}{l}\text { The proposed model has the } \\
\text { potential to help with treatment } \\
\text { planning by improving the efficiency } \\
\text { and consistency of CTVPI } \\
\text { segmentation. }\end{array}$ & $\begin{array}{l}\text { I. Insufficient training data. } \\
\text { 2. Delineation variability between } \\
\text { practitioners. } \\
\text { 3. MRI images were not used for } \\
\text { training the model. }\end{array}$ \\
\hline $\begin{array}{l}\text { Yang et al } \\
(2020)^{28} \\
\text { (China) }\end{array}$ & $\begin{array}{c}\text { NPC } \\
\text { II38 } \\
\text { (MRI images) }\end{array}$ & $\begin{array}{l}\text { Deep learning } \\
\text { (Prognosis) }\end{array}$ & $\begin{array}{l}\text { I. Prediction: } \\
\text { Resnet }\end{array}$ & $\begin{array}{l}\text { To evaluate an } \\
\text { automatic } T \text { staging } \\
\text { system that requires no } \\
\text { additional annotation }\end{array}$ & $\begin{array}{l}\text { I. Prognostic performance } \\
\text { a. AUC: } 0.943 \\
\text { b. Sensitivity: } 0.882 \\
\text { c. Specificity: } 0.880 \\
\text { d. Accuracy: } 0.7559 \text { [Cl: } 0.7151- \\
0.7967] \\
\text { e. C-index } \\
\text { - OS: } 0.652 \text { [CI: } 0.567-0.737] \\
\text { - PFS: } 0.612 \text { [Cl: } 0.537-0.686]\end{array}$ & $\begin{array}{l}\text { The proposed model had a similar performance to } \\
\text { the TNM staging system }\end{array}$ & $\begin{array}{l}\text { The model had a good prognostic } \\
\text { performance in fully automated } T \\
\text { staging of NPC. }\end{array}$ & $\begin{array}{l}\text { 1. Some imaging information may } \\
\text { be ignored as contrast-enhanced- } \\
\text { TI weighted images in the coronal } \\
\text { plane and TI weighted images in } \\
\text { the sagittal plane were not } \\
\text { included in the model } \\
\text { construction. } \\
\text { 2. The generalizability was } \\
\text { unknown as the model was not } \\
\text { externally verified. }\end{array}$ \\
\hline $\begin{array}{l}\text { Yang et al } \\
(2020)^{67} \\
\text { (China) }\end{array}$ & $\begin{array}{c}\text { NPC } \\
147 \\
\text { (CT images) }\end{array}$ & $\begin{array}{l}\text { Deep learning } \\
\text { (Auto- } \\
\text { contouring) }\end{array}$ & $\begin{array}{l}\text { I. Feature } \\
\text { extraction: } \\
\text { Based on 3D U-net }\end{array}$ & $\begin{array}{l}\text { To investigate the } \\
\text { segmentation accuracy } \\
\text { of OARs }\end{array}$ & $\begin{array}{l}\text { I. Segmentation performance } \\
\text { - DSC: } 0.62 \pm 0.02 \\
\text { - HD: } 3.4 \mathrm{~mm} \pm 1.0 \mathrm{~mm}\end{array}$ & $\begin{array}{l}\text { There was no statistical significance between the } \\
\text { results obtained from the proposed model and } \\
\text { manual contouring of the OARs except for the } \\
\text { optic nerves and chiasm. }\end{array}$ & $\begin{array}{l}\text { The developed model can be used } \\
\text { for auto-contouring of OARs. }\end{array}$ & N/A \\
\hline $\begin{array}{l}\text { Zhang et al } \\
(2020)^{58} \\
\text { (China) }\end{array}$ & $\begin{array}{c}\text { NPC } \\
242 \\
\text { (MRI images) }\end{array}$ & $\begin{array}{l}\text { Machine } \\
\text { learning } \\
\text { (Radiation- } \\
\text { induced injury } \\
\text { diagnosis) }\end{array}$ & $\begin{array}{l}\text { I. Radiomic } \\
\text { analysis: } \\
\text { RF }\end{array}$ & $\begin{array}{l}\text { To develop a model for } \\
\text { early detection of } \\
\text { radiation-induced } \\
\text { temporal lobe injury }\end{array}$ & $\begin{array}{l}\text { I. Predictive performance } \\
\text { - AUC: } 0.830 \text { [CI: } 0.823-0.837]\end{array}$ & $\begin{array}{l}\text { The use of texture features in feature selection } \\
\text { improved the performance of the prediction model. }\end{array}$ & $\begin{array}{l}\text { The developed models have the } \\
\text { potential to support in providing } \\
\text { early detection and taking } \\
\text { preventive measures against } \\
\text { radiation-induced temporal lobe } \\
\text { injury. }\end{array}$ & $\begin{array}{l}\text { 1. Insufficient sample size. } \\
\text { 2. 3D-conformal radiotherapy was } \\
\text { performed on NPC patients } \\
\text { instead of IMRT. } \\
\text { 3. Limited generalizability as it was } \\
\text { a single institution study. }\end{array}$ \\
\hline $\begin{array}{l}\text { Zhang et al } \\
(2020)^{36} \\
\text { (China) }\end{array}$ & $\begin{array}{c}\text { NPC } \\
220 \\
\text { (WSIs, MRI images } \\
\text { and } \\
\text { clinicopathological } \\
\text { data) }\end{array}$ & $\begin{array}{l}\text { Deep learning } \\
\text { (Prognosis) }\end{array}$ & $\begin{array}{l}\text { I. Prediction: } \\
\text { Resnet-18 and } \\
\text { DeepSurv }\end{array}$ & $\begin{array}{l}\text { To explore the use of } \\
\text { magnetic resonance } \\
\text { imaging and } \\
\text { microscopic whole- } \\
\text { slide images to improve } \\
\text { the prognosis of model }\end{array}$ & $\begin{array}{l}\text { 1. Prognostic performance } \\
\text { - C-index: } 0.834 \text { [0.736-0.932] }\end{array}$ & $\begin{array}{l}\text { The established nomogram had a much higher } \\
\text { performance compared to the clinical model. }\end{array}$ & $\begin{array}{l}\text { The developed multi-scale } \\
\text { nomogram has the potential to be a } \\
\text { non-invasive, cost-effective tool for } \\
\text { assisting in individualized treatment } \\
\text { and decision making on NPC. }\end{array}$ & $\begin{array}{l}\text { 1. The study was retrospective } \\
\text { and the sample size was relatively } \\
\text { small } \\
\text { 2. A molecular profile was not } \\
\text { used in the model. } \\
\text { 3. The subjects in the cohorts } \\
\text { were all Chinese, hence, the } \\
\text { generalizability needs to be } \\
\text { verified }\end{array}$ \\
\hline
\end{tabular}




\begin{tabular}{|c|c|c|c|c|c|c|c|c|}
\hline $\begin{array}{l}\text { Zhao et al } \\
(2020)^{35} \\
\text { (China) }\end{array}$ & $\begin{array}{l}\text { NPC } \\
123 \\
\text { (MRI images, } \\
\text { clinicopathological } \\
\text { and plasma EBV } \\
\text { DNA data) }\end{array}$ & $\begin{array}{l}\text { Machine } \\
\text { learning } \\
\text { (Prognosis) }\end{array}$ & $\begin{array}{l}\text { I. Prediction: } \\
\text { SVM }\end{array}$ & $\begin{array}{l}\text { To investigate an MRI- } \\
\text { based radiomics } \\
\text { nomogram in } \\
\text { predicting induction } \\
\text { chemotherapy } \\
\text { response and survival }\end{array}$ & $\begin{array}{l}\text { I. Prediction performance } \\
\text { - AUC: } 0.8725 \\
\text { - Accuracy: } 0.8696 \\
\text { - PPV: } 71.43 \% \\
\text { - NPV: } 93.75 \%\end{array}$ & $\begin{array}{l}\text { The proposed nomogram had a better } \\
\text { performance than the clinical nomogram. }\end{array}$ & $\begin{array}{l}\text { The constructed nomogram could } \\
\text { be used for personalized risk } \\
\text { stratification and for treating NPC } \\
\text { patients that received induction } \\
\text { chemotherapy. }\end{array}$ & $\begin{array}{l}\text { I. Small sample size due to the } \\
\text { strict inclusion criteria. } \\
\text { 2. Limited generalizability due to } \\
\text { the single institutional nature of } \\
\text { the study } \\
\text { 3. The induction chemotherapy } \\
\text { response evaluation had a lower } \\
\text { accuracy as the assessment was } \\
\text { based only on anatomical MRI } \\
\text { imaging. }\end{array}$ \\
\hline $\begin{array}{l}\text { Zhong et al } \\
(2020)^{29} \\
\text { (China) }\end{array}$ & $\begin{array}{c}\text { NPC } \\
638 \\
\text { (MRI images, } \\
\text { clinicopathological } \\
\text { and plasma EBV } \\
\text { DNA data) }\end{array}$ & $\begin{array}{l}\text { Deep learning } \\
\text { (Prognosis) }\end{array}$ & $\begin{array}{l}\text { I. Prediction: } \\
\text { ResNeXt }\end{array}$ & $\begin{array}{l}\text { To predict the survival } \\
\text { of stage T3NIMO NPC } \\
\text { patients treated with } \\
\text { induction } \\
\text { chemotherapy and } \\
\text { concurrent } \\
\text { chemoradiotherapy }\end{array}$ & $\begin{array}{l}\text { I. Model performance } \\
\text { - C-index: } 0.788 \text { [Cl: } 0.695-0.882]\end{array}$ & $\begin{array}{l}\text { The DL-based radiomics model had a higher } \\
\text { predictive performance than the clinical model. }\end{array}$ & $\begin{array}{l}\text { It has the potential to be a useful } \\
\text { non-invasive tool for risk } \\
\text { stratification and prognostic } \\
\text { prediction }\end{array}$ & $\begin{array}{l}\text { 1. Only the basilar region was } \\
\text { used for analysis, while } \\
\text { nasopharyngeal and other regions } \\
\text { were not considered. } \\
\text { 2. Only T3NIMO patients were } \\
\text { considered }\end{array}$ \\
\hline $\begin{array}{l}\text { Bai et al } \\
(2021)^{30} \\
\text { (China) }\end{array}$ & $\begin{array}{c}\text { NPC } \\
60 \\
\text { (CT images) }\end{array}$ & $\begin{array}{l}\text { Deep learning } \\
\text { (Auto- } \\
\text { contouring) }\end{array}$ & $\begin{array}{l}\text { I. Segmentation: } \\
\text { ResNeXt U-net }\end{array}$ & $\begin{array}{l}\text { To use computed } \\
\text { tomography for the } \\
\text { segmentation of NPC }\end{array}$ & $\begin{array}{l}\text { I. Segmentation performance } \\
\text { - DSC: } 0.6288 \pm 0.0812 \\
-95 \mathrm{HD}: 6.07 \mathrm{~mm} \pm 2.53 \mathrm{~mm} \\
\text { - FI-score: } 0.6615\end{array}$ & $\begin{array}{l}\text { The developed DL algorithm had a significantly } \\
\text { better performance than three existing DL models }\end{array}$ & $\begin{array}{l}\text { An NPC-seg algorithm was } \\
\text { developed and won 9th place on the } \\
\text { StructSeg } \\
2019 \text { Challenge leader-board }\end{array}$ & N/A \\
\hline $\begin{array}{l}\text { Cai et al } \\
(2021)^{31} \\
\text { (China) }\end{array}$ & $\begin{array}{c}\text { NPC } \\
251 \\
\text { (MRI images and } \\
\text { clinicopathological } \\
\text { data) }\end{array}$ & $\begin{array}{l}\text { Deep learning } \\
\text { (Auto- } \\
\text { contouring) }\end{array}$ & $\begin{array}{l}\text { I. Segmentation: } \\
\text { Attention U-net } \\
\text { with T-channel } \\
\text { module }\end{array}$ & $\begin{array}{l}\text { To use image and } \\
\text { T-staging information } \\
\text { to improve NPC tumor } \\
\text { delineation accuracy }\end{array}$ & $\begin{array}{l}\text { I. Segmentation performance } \\
\text { - DSC: } 0.845 \text { [Cl: } 0.791-0.879] \\
\text { - ASSD: } 0.533 \mathrm{~mm}[\mathrm{Cl}: 0.174-1.254]\end{array}$ & $\begin{array}{l}\text { Having the attention module and T-channel } \\
\text { improved the effectiveness of the model. The } \\
\text { proposed model had the best performance over } \\
\text { four other state-of-The-art methods. }\end{array}$ & $\begin{array}{l}\text { Integrating both the attention and } \\
\text { the T-channel module can improve } \\
\text { the delineation performance of a } \\
\text { model substantially }\end{array}$ & $\begin{array}{l}\text { 1. Small batch size due to GPU } \\
\text { memory limitation } \\
\text { 2. Smaller number of epochs to } \\
\text { reduce the training time. } \\
\text { 3. The proportion of T-channel } \\
\text { against the input volume is small. }\end{array}$ \\
\hline $\begin{array}{l}\text { Tang et al } \\
(2021)^{32} \text { (China } \\
\text { and Australia) }\end{array}$ & $\begin{array}{c}\text { NPC } \\
95 \\
\text { (MRI images) }\end{array}$ & $\begin{array}{l}\text { Deep learning } \\
\text { (Auto- } \\
\text { contouring) }\end{array}$ & $\begin{array}{l}\text { I. Segmentation: } \\
\text { DA-DSU-net }\end{array}$ & $\begin{array}{l}\text { To develop a model for } \\
\text { NPC segmentation } \\
\text { using MRI }\end{array}$ & $\begin{array}{l}\text { I. Segmentation performance } \\
\text { - DSC: } 0.8050 \\
\text { - ASSD: } 0.8021 \mathrm{~mm} \\
\text { - Prevent match: } 0.8026 \\
\text { - Correspondence ratio: } 0.7065\end{array}$ & $\begin{array}{l}\text { The developed network had a higher performance } \\
\text { than three other segmentation methods }\end{array}$ & $\begin{array}{l}\text { The proposed model can help } \\
\text { clinicians by delineating the tumor in } \\
\text { order to provide accurate staging } \\
\text { and radiotherapy planning of NPC. }\end{array}$ & $\begin{array}{l}\text { 1. Insufficient training data. } \\
\text { 2. The model processes in } 2 \mathrm{D} \\
\text { form. } \\
\text { 3. Multi-modality input was not } \\
\text { used. }\end{array}$ \\
\hline $\begin{array}{l}\text { Wen et al } \\
(2021)^{57} \\
\text { (China) }\end{array}$ & $\begin{array}{c}\text { NPC } \\
8194 \\
\text { (Clinicopathological } \\
\text { and dosimetric data) }\end{array}$ & $\begin{array}{l}\text { Machine } \\
\text { learning } \\
\text { (Radiation- } \\
\text { induced injury } \\
\text { diagnosis) }\end{array}$ & $\begin{array}{l}\text { I. Dosimetric } \\
\text { factors selection: } \\
\text { LASSO, RF; } \\
\text { Stochastic gradient } \\
\text { boosting and SVM }\end{array}$ & $\begin{array}{l}\text { To predict temporal } \\
\text { lobe injury after } \\
\text { intensity-modulated } \\
\text { radiotherapy in NPC }\end{array}$ & $\begin{array}{l}\text { I. Identification of dosimetric factors } \\
\text { associated with temporal lobe injury } \\
\text { incidence } \\
\text { - AUC: } 0.818 \\
\text { - C-index: } 0.775 \text { [Cl: } 0.751-0.799] \\
\text { - Spearman correlation matrix }\end{array}$ & $\begin{array}{l}\text { The nomogram that included dosimetric and clinical } \\
\text { factors had a better prediction performance than } \\
\text { the nomogram with only DVH. } \\
\text { D0.5cc was considered the most important } \\
\text { dosimetric factor by LASSO, Stochastic gradient } \\
\text { boosting and SVM. }\end{array}$ & $\begin{array}{l}\text { The proposed method was able to } \\
\text { predict temporal lobe injury } \\
\text { accurately and can be used to help } \\
\text { provide individualized follow-up } \\
\text { management. }\end{array}$ & $\begin{array}{l}\text { 1. Selection bias due to the } \\
\text { retrospective nature of the study. } \\
\text { 2. No external validation due to } \\
\text { the single institutional nature of } \\
\text { the study. } \\
\text { 3. TLl were not grouped by the } \\
\text { severity. }\end{array}$ \\
\hline
\end{tabular}

(Continued) 


\begin{tabular}{|c|c|c|c|c|c|c|c|c|}
\hline $\begin{array}{l}\text { Authors, } \\
\text { Year and } \\
\text { Country }\end{array}$ & $\begin{array}{l}\text { Site, No. of Cases } \\
\text { (Data Type) }\end{array}$ & $\begin{array}{l}\text { Al Subfield } \\
\text { (Application) }\end{array}$ & $\begin{array}{c}\text { Artificial } \\
\text { Intelligence } \\
\text { Methods and its } \\
\text { Application }\end{array}$ & Study Aim & Performance Metric (s) & Results & Conclusion & Limitations \\
\hline $\begin{array}{l}\text { Wong et al } \\
(2021)^{45} \text { (Hong } \\
\text { Kong SAR, } \\
\text { China) }\end{array}$ & $\begin{array}{c}\text { NPC } \\
412 \\
\text { (MRI images) }\end{array}$ & $\begin{array}{l}\text { Deep learning } \\
\text { (Diagnosis) }\end{array}$ & $\begin{array}{l}\text { I. Classification: } \\
\text { Residual Attention } \\
\text { Network }\end{array}$ & $\begin{array}{l}\text { To differentiate early } \\
\text { stage NPC from benign } \\
\text { hyperplasia using T2- } \\
\text { weighted MRI }\end{array}$ & $\begin{array}{l}\text { I. Diagnostic performance } \\
\text { - AUC: } 0.96 \text { [Cl: } 0.94-0.98] \\
\text { - Sensitivity: } 0.924 \text { [CI: } 0.858-0.959] \\
\text { - Specificity: } 0.906 \text { [Cl: } 0.728-0.951] \\
\text { - Accuracy: } 0.915 \\
\text { - PPV: } 0.905 \\
\text { - NPV: } 0.924\end{array}$ & $\begin{array}{l}\text { The CNN obtained a good result in discriminating } \\
\text { NPC and benign hyperplasia. }\end{array}$ & $\begin{array}{l}\text { The proposed fully automatic } \\
\text { network model demonstrated the } \\
\text { prospect of CNN in identifying NPC } \\
\text { at an early stage. }\end{array}$ & $\begin{array}{l}\text { I. There is limited generalizability } \\
\text { as only MRI scans of the head and } \\
\text { neck region with the field of view } \\
\text { centered on the nasopharynx can } \\
\text { be used. } \\
\text { 2. No external validation. } \\
\text { 3. No association between CNN } \\
\text { score and having a nasopharyngeal } \\
\text { biopsy. } \\
\text { 4. Possible chance to include } \\
\text { undetected NPC in patients with } \\
\text { benign hyperplasia. }\end{array}$ \\
\hline $\begin{array}{l}\text { Wong et al } \\
(2021)^{56} \text { (Hong } \\
\text { Kong SAR, } \\
\text { China) }\end{array}$ & $\begin{array}{c}\text { NPC } \\
201 \\
\text { (MRI images) }\end{array}$ & $\begin{array}{l}\text { Deep learning } \\
\text { (Auto- } \\
\text { contouring) }\end{array}$ & $\begin{array}{l}\text { I. Delineation: } \\
\text { U-net }\end{array}$ & $\begin{array}{l}\text { To evaluate the } \\
\text { delineation } \\
\text { performance of a } \\
\text { model using non- } \\
\text { contrast-enhanced MRI }\end{array}$ & $\begin{array}{l}\text { I. Delineation performance } \\
\text { - DSC: } 0.71 \pm 0.09 \\
\text { - ASSD: } 2.1 \mathrm{~mm} \pm 4.8 \mathrm{~mm} \\
-\Delta \text { Primary tumor volume: } 1.0 \\
\pm 12.2 \mathrm{~cm}^{3}\end{array}$ & $\begin{array}{l}\text { The performance of CNN using fs-T2W images } \\
\text { was similar to that of CNNs using contrast- } \\
\text { enhanced-TI weighted and contrast-enhanced-fat- } \\
\text { suppressed-TI weighted images. }\end{array}$ & $\begin{array}{l}\text { Although using contrast-enhanced } \\
\text { sequence for head and neck MRI is } \\
\text { still recommended, when avoiding } \\
\text { use of contrast agent is preferred, } \\
\text { CNN is a potential future option. }\end{array}$ & $\begin{array}{l}\text { I. Limited generalizability to other } \\
\text { CNN architectures due to } \\
\text { variations in tissue contrasts. } \\
\text { 2. Only considered slice-based } \\
\text { algorithms and no other ones. } \\
\text { 3. The study did not take into } \\
\text { account whether CNN } \\
\text { delineation performance could be } \\
\text { affected by different fat- } \\
\text { suppression techniques. }\end{array}$ \\
\hline $\begin{array}{l}\text { Wu et al } \\
(2021)^{34} \\
\text { (China) }\end{array}$ & $\begin{array}{l}\text { NPC and other } \\
\text { types } \\
233 \\
\text { (MRI images) }\end{array}$ & $\begin{array}{l}\text { Machine } \\
\text { learning and } \\
\text { deep learning } \\
\text { (Prognosis) }\end{array}$ & $\begin{array}{l}\text { I. Classification: } \\
\text { Resnet/8 } \\
\text { 2. Feature selection } \\
\text { LASSO }\end{array}$ & $\begin{array}{l}\text { To assess the predicted } \\
\text { value of peritumoral } \\
\text { regions and explore } \\
\text { the effects of different } \\
\text { peritumoral sizes in } \\
\text { learning models }\end{array}$ & $\begin{array}{l}\text { I. Model performance } \\
\text { a) AUC: } 0.660 \text { [Cl: } 0.484-0.837] \\
\text { b) Sensitivity: } 0.344 \text { [Cl: } 0.179-0.508] \\
\text { c) Specificity: } 0.800 \text { [Cl: } 0.598-1.000]\end{array}$ & $\begin{array}{l}\text { Radiomics is more suitable than DL for modelling } \\
\text { peritumors }\end{array}$ & $\begin{array}{l}\text { The peritumoral models, and ML } \\
\text { and } D L \text { helped improved the } \\
\text { prediction performance. }\end{array}$ & $\begin{array}{l}\text { 1. Datasets were small. } \\
\text { 2. Maximum slice of the tumor } \\
\text { was used for analysis directly, but } \\
\text { multi-slices of the tumor were } \\
\text { not. }\end{array}$ \\
\hline $\begin{array}{l}\text { Zhang et al } \\
(2021)^{33} \\
\text { (China) }\end{array}$ & $\begin{array}{l}\text { NPC } \\
252 \\
\text { (MRI images, } \\
\text { clinicopathological } \\
\text { and plasma EBV } \\
\text { DNA data) }\end{array}$ & $\begin{array}{l}\text { Machine } \\
\text { learning and } \\
\text { deep learning } \\
\text { (Prognosis) }\end{array}$ & $\begin{array}{l}\text { I. Prediction: } \\
\text { Residual network } \\
\text { and LR analysis } \\
\text { 2. Feature } \\
\text { selection: } \\
\text { Minimum } \\
\text { redundancy- } \\
\text { maximum } \\
\text { relevance, LASSO } \\
\text { \& Akaike } \\
\text { information } \\
\text { criterion algorithms }\end{array}$ & $\begin{array}{l}\text { To predict DMFS and } \\
\text { to investigate the } \\
\text { influence of additional } \\
\text { chemotherapy to } \\
\text { concurrent } \\
\text { chemoradiotherapy for } \\
\text { different risk groups. }\end{array}$ & $\begin{array}{l}\text { 1. Prediction performance of Distant } \\
\text { metastasis-free survival } \\
\text { a) AUC - DMFS: } 0.808 \text { [Cl: } 0.654- \\
0.962 \text { ] }\end{array}$ & $\begin{array}{l}\text { By integrating DL signature with N stage, EBV DNA } \\
\text { and treatment regimen, the MRI-based combined } \\
\text { model had a better predictive performance than } \\
\text { the DL signature-based, radiomic signature-based } \\
\text { and clinical-based model }\end{array}$ & $\begin{array}{l}\text { The MRI-based combined model } \\
\text { could be used as a complementary } \\
\text { tool for making treatment decisions } \\
\text { by assessing the risk of DMFS in } \\
\text { locoregionally advanced NPC } \\
\text { patients }\end{array}$ & $\begin{array}{l}\text { 1. The value of the deep learning } \\
\text { model and the collected } \\
\text { information were limited. } \\
\text { 2. The repeatability of radiomic } \\
\text { signatures was poor. } \\
\text { 3. The generalizability of the } \\
\text { model is affected due to } \\
\text { difference in scan protocols } \\
\text { between institutions. }\end{array}$ \\
\hline
\end{tabular}

Notes: andicates performance metric presented in graph and not as a numerical value. ${ }^{b}$ Values found in publication.

Abbreviations: NPC, nasopharyngeal carcinoma; MRI, magnetic resonance imaging; SVM, support vector machines; KNN, k-nearest neighbor; ANN, artificial neural network; AUC, area under the receiver operating characteristic curve; ML, machine learning; PET, positron emission tomography; CT, computed tomography; PPV, positive predictive values; NPV, negative predictive values; HD, Hausdorff distance; LR, logistic regression; RF, random forest; C-index, concordance index; CNN, convolutional neural network; IMRT, intensity-modulated radiation therapy; DVH, dose-volume histogram; MAE, mean absolute error; OAR, organ-at-risk; EBV DNA, Epstein-Barr Virus DNA; DL, deep learning; VMAT, volumetric modulated arc therapy; WSI, whole slide image; LASSO, least absolute shrinkage and selection operator; OS, Overall survival; DMFS, distant metastasis-free survival; LRFS, local-region relapse-free survival; AJCC, American Joint Committee on Cancer; PFS, progression-free survival. 
Table 2 Quality Assessment via the QUADAS-2 Tool

\begin{tabular}{|c|c|c|c|c|c|c|c|c|c|}
\hline \multirow{2}{*}{$\begin{array}{l}\text { Authors Publication } \\
\text { Year }\end{array}$} & \multicolumn{4}{|c|}{ Risk of Bias ${ }^{a}$} & \multicolumn{3}{|c|}{ Applicability Concerns ${ }^{a}$} & \multicolumn{2}{|c|}{ At Risk ${ }^{\mathbf{b}}$} \\
\hline & $\begin{array}{l}\text { Patients } \\
\text { Selection }\end{array}$ & $\begin{array}{c}\text { Index } \\
\text { Test }\end{array}$ & $\begin{array}{l}\text { Reference } \\
\text { Standard }\end{array}$ & $\begin{array}{c}\text { Flow } \\
\text { and } \\
\text { Timing }\end{array}$ & $\begin{array}{l}\text { Patients } \\
\text { Selection }\end{array}$ & $\begin{array}{c}\text { Index } \\
\text { Test }\end{array}$ & $\begin{array}{l}\text { Reference } \\
\text { Standard }\end{array}$ & $\begin{array}{c}\text { Risk } \\
\text { of } \\
\text { Bias }\end{array}$ & Applicability \\
\hline Wang et al $(2010)^{10}$ & $?$ & $\checkmark$ & $\checkmark$ & $\checkmark$ & $\checkmark$ & $\checkmark$ & $\checkmark$ & Yes & No \\
\hline Aussem et al $(2012)^{\prime \prime}$ & $?$ & $\checkmark$ & $\checkmark$ & $\checkmark$ & $\checkmark$ & $\checkmark$ & $\checkmark$ & Yes & No \\
\hline $\begin{array}{l}\text { Kumdee, Bhongmakapat } \\
\text { and Ritthipravat }(2012)^{12}\end{array}$ & $\checkmark$ & $\checkmark$ & $\checkmark$ & $\checkmark$ & $\checkmark$ & $\checkmark$ & $\checkmark$ & No & No \\
\hline $\begin{array}{l}\text { Ritthipravat, Kumdee, and } \\
\text { Bhongmakapat }(2013)^{13}\end{array}$ & $\checkmark$ & $\checkmark$ & $\checkmark$ & $\checkmark$ & $\checkmark$ & $\checkmark$ & $\checkmark$ & No & No \\
\hline Zhu and Kan $(2014)^{44}$ & $\checkmark$ & $\checkmark$ & $\checkmark$ & $\checkmark$ & $\checkmark$ & $\checkmark$ & $\checkmark$ & No & No \\
\hline Jiang et al $(2016)^{14}$ & $?$ & $\checkmark$ & $\checkmark$ & $\checkmark$ & $\checkmark$ & $\checkmark$ & $\checkmark$ & Yes & No \\
\hline Liu et al $(2016)^{43}$ & $\checkmark$ & $\checkmark$ & $\checkmark$ & $?$ & $\checkmark$ & $\checkmark$ & $\checkmark$ & Yes & No \\
\hline Wang et al $(2016)^{63}$ & $?$ & $\checkmark$ & $\checkmark$ & $\checkmark$ & $\checkmark$ & $\checkmark$ & $\checkmark$ & Yes & No \\
\hline Men et al $(2017)^{55}$ & $\checkmark$ & $\checkmark$ & $\checkmark$ & $?$ & $\checkmark$ & $\checkmark$ & $\checkmark$ & Yes & No \\
\hline Mohammed et al $(2017)^{54}$ & $?$ & $\checkmark$ & $\checkmark$ & $\checkmark$ & $\checkmark$ & $\checkmark$ & $\checkmark$ & Yes & No \\
\hline Zhang et al $(2017)^{42}$ & $\checkmark$ & $\checkmark$ & $\checkmark$ & $\checkmark$ & $\checkmark$ & $\checkmark$ & $\checkmark$ & No & No \\
\hline Zhang et al $(2017)^{41}$ & $\checkmark$ & $\checkmark$ & $\checkmark$ & $?$ & $\checkmark$ & $\checkmark$ & $\checkmark$ & Yes & No \\
\hline Li et al $(2018)^{15}$ & $\checkmark$ & $\checkmark$ & $\checkmark$ & $\checkmark$ & $\checkmark$ & $\checkmark$ & $\checkmark$ & No & No \\
\hline Mohammed et al $(2018)^{62}$ & $?$ & $\checkmark$ & $\checkmark$ & $\checkmark$ & $\checkmark$ & $\checkmark$ & $\checkmark$ & Yes & No \\
\hline Mohammed et al $(2018)^{16}$ & ? & $\checkmark$ & $\checkmark$ & $\checkmark$ & $\checkmark$ & $\checkmark$ & $\checkmark$ & Yes & No \\
\hline Du et al $(2019)^{40}$ & $\checkmark$ & $\checkmark$ & $\checkmark$ & $\checkmark$ & $\checkmark$ & $\checkmark$ & $\checkmark$ & No & No \\
\hline Jiao et al $(2019)^{66}$ & $\checkmark$ & $\checkmark$ & $?$ & $\checkmark$ & $\checkmark$ & $\checkmark$ & $\checkmark$ & Yes & No \\
\hline Jing et al $(2019)^{17}$ & $\checkmark$ & $\checkmark$ & $\checkmark$ & $\checkmark$ & $\checkmark$ & $\checkmark$ & $\checkmark$ & No & No \\
\hline Li et al $(2019)^{53}$ & $\checkmark$ & $\checkmark$ & $\checkmark$ & $\checkmark$ & $\checkmark$ & $\checkmark$ & $\checkmark$ & No & No \\
\hline Liang et al $(2019)^{52}$ & $\checkmark$ & $\checkmark$ & $\checkmark$ & $\checkmark$ & $\checkmark$ & $\checkmark$ & $\checkmark$ & No & No \\
\hline Lin et al $(2019)^{51}$ & $\checkmark$ & $\checkmark$ & $\checkmark$ & $\checkmark$ & $\checkmark$ & $\checkmark$ & $\checkmark$ & No & No \\
\hline Liu et al $(2019)^{65}$ & $\checkmark$ & $\checkmark$ & $?$ & $\checkmark$ & $\checkmark$ & $\checkmark$ & $\checkmark$ & Yes & No \\
\hline Ma et al $(2019)^{18}$ & $?$ & $\checkmark$ & $\checkmark$ & $\checkmark$ & $\checkmark$ & $\checkmark$ & $\checkmark$ & Yes & No \\
\hline Peng et al $(2019)^{19}$ & $\checkmark$ & $\checkmark$ & $\checkmark$ & $\checkmark$ & $\checkmark$ & $\checkmark$ & $\checkmark$ & No & No \\
\hline Rehioui et al $(2019)^{20}$ & $\checkmark$ & $\checkmark$ & $\checkmark$ & $?$ & $\checkmark$ & $\checkmark$ & $\checkmark$ & Yes & No \\
\hline Zhong et al $(2019)^{50}$ & ? & $\checkmark$ & $\checkmark$ & $\checkmark$ & $\checkmark$ & $\checkmark$ & $\checkmark$ & Yes & No \\
\hline Zou et al $(2019)^{21}$ & ? & $\checkmark$ & $\checkmark$ & $\checkmark$ & $\checkmark$ & $\checkmark$ & $\checkmark$ & Yes & No \\
\hline Abd Ghani et al $(2020)^{61}$ & ? & $\checkmark$ & $\checkmark$ & $\checkmark$ & $\checkmark$ & $\checkmark$ & $\checkmark$ & Yes & No \\
\hline Bai et al $(2020)^{64}$ & $\checkmark$ & $\checkmark$ & $\checkmark$ & $\checkmark$ & $\checkmark$ & $\checkmark$ & $\checkmark$ & No & No \\
\hline Chen et al $(2020)^{22}$ & $?$ & $\checkmark$ & $\checkmark$ & $\checkmark$ & $\checkmark$ & $\checkmark$ & $\checkmark$ & Yes & No \\
\hline
\end{tabular}


Table 2 (Continued).

\begin{tabular}{|c|c|c|c|c|c|c|c|c|c|}
\hline \multirow{2}{*}{$\begin{array}{l}\text { Authors Publication } \\
\text { Year }\end{array}$} & \multicolumn{4}{|c|}{ Risk of Bias ${ }^{a}$} & \multicolumn{3}{|c|}{ Applicability Concerns ${ }^{a}$} & \multicolumn{2}{|c|}{ At Risk ${ }^{b}$} \\
\hline & $\begin{array}{l}\text { Patients } \\
\text { Selection }\end{array}$ & $\begin{array}{l}\text { Index } \\
\text { Test }\end{array}$ & $\begin{array}{l}\text { Reference } \\
\text { Standard }\end{array}$ & $\begin{array}{c}\text { Flow } \\
\text { and } \\
\text { Timing }\end{array}$ & $\begin{array}{l}\text { Patients } \\
\text { Selection }\end{array}$ & $\begin{array}{c}\text { Index } \\
\text { Test }\end{array}$ & $\begin{array}{c}\text { Reference } \\
\text { Standard }\end{array}$ & $\begin{array}{c}\text { Risk } \\
\text { of } \\
\text { Bias }\end{array}$ & Applicability \\
\hline Chen et al $(2020)^{68}$ & $\checkmark$ & $\checkmark$ & $\checkmark$ & $\checkmark$ & $\checkmark$ & $\checkmark$ & $\checkmark$ & No & No \\
\hline Chuang et al $(2020)^{23}$ & $\checkmark$ & $\checkmark$ & $\checkmark$ & $?$ & $\checkmark$ & $\checkmark$ & $\checkmark$ & Yes & No \\
\hline Cui et al $(2020)^{39}$ & $\checkmark$ & $\checkmark$ & $\checkmark$ & $\checkmark$ & $\checkmark$ & $?$ & $\checkmark$ & No & Yes \\
\hline Diao et al $(2020)^{60}$ & $\checkmark$ & $\checkmark$ & $\checkmark$ & $?$ & $\checkmark$ & $\checkmark$ & $\checkmark$ & Yes & No \\
\hline Du et al $(2020)^{59}$ & $\checkmark$ & $\checkmark$ & $\checkmark$ & $\checkmark$ & $\checkmark$ & $\checkmark$ & $\checkmark$ & No & No \\
\hline Guo et al $(2020)^{24}$ & $?$ & $\checkmark$ & $\checkmark$ & $\checkmark$ & $\checkmark$ & $\checkmark$ & $\checkmark$ & Yes & No \\
\hline Jing et al $(2020)^{25}$ & $\checkmark$ & $\checkmark$ & $\checkmark$ & $\checkmark$ & $\checkmark$ & $\checkmark$ & $\checkmark$ & No & No \\
\hline Ke et al $(2020)^{49}$ & $\checkmark$ & $\checkmark$ & $\checkmark$ & $\checkmark$ & $\checkmark$ & $\checkmark$ & $\checkmark$ & No & No \\
\hline Liu et al $(2020)^{38}$ & $?$ & $\checkmark$ & $\checkmark$ & $\checkmark$ & $\checkmark$ & $\checkmark$ & $\checkmark$ & Yes & No \\
\hline Men et al $(2020)^{47}$ & $?$ & $\checkmark$ & $\checkmark$ & $\checkmark$ & $\checkmark$ & $\checkmark$ & $\checkmark$ & Yes & No \\
\hline Mohammed et al $(2020)^{26}$ & $?$ & $\checkmark$ & $\checkmark$ & $\checkmark$ & $\checkmark$ & $\checkmark$ & $\checkmark$ & Yes & No \\
\hline Wang et al $(2020)^{27}$ & $\checkmark$ & $\checkmark$ & $\checkmark$ & $\checkmark$ & $\checkmark$ & $\checkmark$ & $\checkmark$ & No & No \\
\hline Wang et al $(2020)^{48}$ & $\checkmark$ & $\checkmark$ & $\checkmark$ & $?$ & $\checkmark$ & $\checkmark$ & $\checkmark$ & Yes & No \\
\hline Xie et al $(2020)^{37}$ & $\checkmark$ & $\checkmark$ & $\checkmark$ & $\checkmark$ & $\checkmark$ & $\checkmark$ & $\checkmark$ & No & No \\
\hline Xue et al $(2020)^{69}$ & $\checkmark$ & $\checkmark$ & $\checkmark$ & $\checkmark$ & $\checkmark$ & $\checkmark$ & $\checkmark$ & No & No \\
\hline Xue et al $(2020)^{46}$ & $\checkmark$ & $\checkmark$ & $\checkmark$ & ? & $\checkmark$ & $\checkmark$ & $\checkmark$ & Yes & No \\
\hline Yang et al $(2020)^{28}$ & $\checkmark$ & $\checkmark$ & $\checkmark$ & $\checkmark$ & $\checkmark$ & $\checkmark$ & $\checkmark$ & No & No \\
\hline Yang et al $(2020)^{67}$ & $?$ & $\checkmark$ & $\checkmark$ & $\checkmark$ & $\checkmark$ & $\checkmark$ & $\checkmark$ & Yes & No \\
\hline Zhang et al $(2020)^{58}$ & $\checkmark$ & $\checkmark$ & $\checkmark$ & $\checkmark$ & $\checkmark$ & $\checkmark$ & $\checkmark$ & No & No \\
\hline Zhang et al $(2020)^{36}$ & $\checkmark$ & $\checkmark$ & $\checkmark$ & $\checkmark$ & $\checkmark$ & $\checkmark$ & $\checkmark$ & No & No \\
\hline Zhao et al $(2020)^{35}$ & $\checkmark$ & $\checkmark$ & $\checkmark$ & $?$ & $\checkmark$ & $\checkmark$ & $\checkmark$ & Yes & No \\
\hline Zhong et al $(2020)^{29}$ & $\checkmark$ & $\checkmark$ & $\checkmark$ & $\checkmark$ & $\checkmark$ & $\checkmark$ & $\checkmark$ & No & No \\
\hline Bai et al $(2021)^{30}$ & $\checkmark$ & $\checkmark$ & $\checkmark$ & $?$ & $\checkmark$ & $\checkmark$ & $\checkmark$ & Yes & No \\
\hline Cai et al $(202 I)^{3 !}$ & $\checkmark$ & $\checkmark$ & $x$ & $\checkmark$ & $\checkmark$ & $\checkmark$ & $\checkmark$ & Yes & No \\
\hline Tang et al $(2021)^{32}$ & $?$ & $\checkmark$ & $\checkmark$ & $\checkmark$ & $\checkmark$ & $\checkmark$ & $\checkmark$ & Yes & No \\
\hline Wen et al $(202 I)^{57}$ & $\checkmark$ & $\checkmark$ & $?$ & $\checkmark$ & $\checkmark$ & $\checkmark$ & $\checkmark$ & Yes & No \\
\hline Wong et al $(202 \mathrm{I})^{45}$ & $\checkmark$ & $\checkmark$ & $\checkmark$ & $\checkmark$ & $\checkmark$ & $\checkmark$ & $\checkmark$ & No & No \\
\hline Wong et al $(202 \mathrm{I})^{56}$ & $\checkmark$ & $\checkmark$ & $x$ & $\checkmark$ & $\checkmark$ & $\checkmark$ & $\checkmark$ & Yes & No \\
\hline$W u$ et al $(202 I)^{34}$ & $\checkmark$ & $\checkmark$ & $\checkmark$ & $\checkmark$ & $\checkmark$ & $\checkmark$ & $\checkmark$ & No & No \\
\hline Zhang et al $(2021)^{33}$ & $\checkmark$ & $\checkmark$ & $\checkmark$ & $?$ & $\checkmark$ & $\checkmark$ & $\checkmark$ & Yes & No \\
\hline
\end{tabular}

Notes: a A check mark $(\checkmark)$ refers to passing (ie, absence of risk) of the criteria; a cross mark (X) refers to not passing (ie, presence of risk) of the criteria; and a question mark (?) refers to missing information to assess the criteria. "The domain "risk of bias" and "applicability" were considered as no bias (ie, "No") if passing all of the corresponding criteria (ie, all $\checkmark$ ); and were considered as having bias (ie, "Yes") if not passing any of the corresponding criteria (ie, at least one ? Or X). 
Table 3 Quality Assessment Guidelines

\begin{tabular}{|c|c|c|}
\hline $\begin{array}{l}\text { Article } \\
\text { Sections }\end{array}$ & Parameters & Explanation \\
\hline \multirow{2}{*}{$\begin{array}{l}\text { Title and } \\
\text { Abstract }\end{array}$} & Title (Nature of study) & Introduce predictive model \\
\hline & $\begin{array}{l}\text { Abstract (Structured } \\
\text { summary) }\end{array}$ & $\begin{array}{l}\text { Include background, objectives, data sources, performance metrics of predictive models and } \\
\text { conclusion about model value }\end{array}$ \\
\hline \multirow[t]{2}{*}{ Introduction } & Rationale & Define the clinical goal, and review the current practice and performance of existing models \\
\hline & Objectives & Identify how the proposed method can benefit the clinical target \\
\hline \multirow[t]{4}{*}{ Method } & Describe the setting & Describe the data source, sample size, year and duration of the data \\
\hline & $\begin{array}{l}\text { Define the prediction } \\
\text { problem }\end{array}$ & $\begin{array}{l}\text { Define the nature of the study (retrospective/prospective), model function (prognosis, diagnosis, } \\
\text { etc.) and performance metrics }\end{array}$ \\
\hline & $\begin{array}{l}\text { Prepare data for model } \\
\text { building }\end{array}$ & $\begin{array}{l}\text { Describe the inclusion and exclusion criteria of the data, data pre-processing method, performance } \\
\text { metrics for validation, and define the training and testing set. External validation is recommended }\end{array}$ \\
\hline & Build the predictive model & $\begin{array}{l}\text { Describe how the model was built including Al modelling techniques used (eg random forest, ANN, } \\
\text { CNN) }\end{array}$ \\
\hline Results & $\begin{array}{l}\text { Report the final model and } \\
\text { performance }\end{array}$ & $\begin{array}{l}\text { Reports the performance of the final proposed model, comparison with other models and human } \\
\text { performance. It is recommended to include confidence intervals }\end{array}$ \\
\hline \multirow[t]{2}{*}{ Discussion } & Clinical implications & Discuss any significant findings \\
\hline & Limitations of the model & Discuss any possible limitations found \\
\hline Conclusion & & Discuss the clinical benefit of the model and summarize the result and findings \\
\hline
\end{tabular}

Note: Data from the guideline of Luo et al. ${ }^{8}$

reporting ML predictive models from Luo et al and Alabi et al (Table 3). ${ }^{8,9}$ The guideline was summarised, and a mark was given for each guideline topic followed. The threshold was set at half of the maximum marks, and the score was presented in Table 4 .

\section{Results}

\section{Database Search}

The selection process was performed using the PRISMA flow diagram in Figure 1. 304 papers were retrieved from the three databases. After 148 duplicates were removed, one inaccessible article was rejected. The papers not meeting the inclusion $(n=59)$ and exclusion $(n=20)$ criteria were also filtered out. Moreover, two additional studies found in literature reviews were included after removing one for being duplicated and another that did not meet the exclusion criteria. Finally, 78 papers were then assessed for quality (Figure 1).

\section{Quality Assessment}

18 papers failed due to having more than one section with a "high" or "unclear" rating, leaving 60 studies to be further evaluated. The QUADAS-2 tool showed that $48.3 \%$ of articles showed an overall low risk of bias, while $98.3 \%$ of them had a low concern regarding applicability (Table 2).

An additional evaluation was performed based on Table 3, which was adapted from the guidelines by Luo et al and the modified version from Alabi et $\mathrm{al}^{8,9}$ Of the 60 relevant studies, 52 of them scored greater than $70 \%$ (Table 4). It should also be noted that 23 papers included the evaluation criteria items but did not fully follow the structure of the proposed guidelines. ${ }^{10-32}$ However, this only affects the ease of reading and extracting information from the articles, but not the content and quality of the papers.

\section{Characteristics of Relevant Studies}

The characteristics of the 7articles finally included in the current study were shown in Table 1. The articles were published in either English $(n=57)^{10-66}$ or Chinese $(n=3) ;{ }^{67-69} 3$ studies examined sites other than the NPC. ${ }^{10,17,34}$ 
Table 4 Quality Scores of the Finalized Articles

\begin{tabular}{|c|c|c|c|c|c|c|c|c|c|c|c|c|}
\hline Studies & Title & Abstract & Rationale & Objectives & $\begin{array}{c}\text { Setting } \\
\text { Description }\end{array}$ & $\begin{array}{l}\text { Problem } \\
\text { Definition }\end{array}$ & $\begin{array}{c}\text { Data } \\
\text { Preparation }\end{array}$ & $\begin{array}{l}\text { Build } \\
\text { Model }\end{array}$ & $\begin{array}{c}\text { Report } \\
\text { Performance }\end{array}$ & $\begin{array}{c}\text { Clinical } \\
\text { Implications }\end{array}$ & Limitations & $\begin{array}{c}\text { Scores } \\
(\%)\end{array}$ \\
\hline Wang et al $(2010)^{10}$ & $\checkmark$ & $x$ & $\checkmark$ & $\checkmark$ & $x$ & $\checkmark$ & $\checkmark$ & $\checkmark$ & $\checkmark$ & $\checkmark$ & $x$ & $73 \%$ \\
\hline Aussem et al (2012) ${ }^{\prime \prime}$ & $\checkmark$ & $x$ & $\checkmark$ & $\checkmark$ & $x$ & $\checkmark$ & $\checkmark$ & $\checkmark$ & $\checkmark$ & $\checkmark$ & $x$ & $73 \%$ \\
\hline $\begin{array}{l}\text { Kumdee, Bhongmakapat and } \\
\text { Ritthipravat }(2012)^{12}\end{array}$ & $\checkmark$ & $\checkmark$ & $\checkmark$ & $x$ & $\checkmark$ & $\checkmark$ & $\checkmark$ & $\checkmark$ & $\checkmark$ & $\checkmark$ & $x$ & $82 \%$ \\
\hline $\begin{array}{l}\text { Ritthipravat, Kumdee and } \\
\text { Bhongmakapat }(2013)^{13}\end{array}$ & $\checkmark$ & $x$ & $\checkmark$ & $x$ & $\checkmark$ & $\checkmark$ & $\checkmark$ & $\checkmark$ & $\checkmark$ & $\checkmark$ & $x$ & $73 \%$ \\
\hline Zhu and Kan $(2014)^{44}$ & $\checkmark$ & $\checkmark$ & $\checkmark$ & $\checkmark$ & $x$ & $\checkmark$ & $\checkmark$ & $\checkmark$ & $\checkmark$ & $\checkmark$ & $x$ & $82 \%$ \\
\hline Jiang et al $(2016)^{14}$ & $\checkmark$ & $\checkmark$ & $\checkmark$ & $\checkmark$ & $\checkmark$ & $\checkmark$ & $\checkmark$ & $\checkmark$ & $x$ & $x$ & $\checkmark$ & $82 \%$ \\
\hline Liu et al $(2016)^{43}$ & $\checkmark$ & $\checkmark$ & $\checkmark$ & $\checkmark$ & $\checkmark$ & $\checkmark$ & $\checkmark$ & $\checkmark$ & $\checkmark$ & $\checkmark$ & $\checkmark$ & $100 \%$ \\
\hline Wang et al $(2016)^{63}$ & $x$ & $\checkmark$ & $\checkmark$ & $\checkmark$ & $x$ & $\checkmark$ & $\checkmark$ & $\checkmark$ & $\checkmark$ & $\checkmark$ & $\checkmark$ & $82 \%$ \\
\hline Men et al $(2017)^{55}$ & $\checkmark$ & $\checkmark$ & $\checkmark$ & $\checkmark$ & $\checkmark$ & $\checkmark$ & $\checkmark$ & $\checkmark$ & $\checkmark$ & $\checkmark$ & $\checkmark$ & $100 \%$ \\
\hline Mohammed et al $(2017)^{54}$ & $\checkmark$ & $x$ & $\checkmark$ & $\checkmark$ & $x$ & $\checkmark$ & $\checkmark$ & $\checkmark$ & $\checkmark$ & $\checkmark$ & $\checkmark$ & $82 \%$ \\
\hline Zhang et al $(2017)^{42}$ & $\checkmark$ & $\checkmark$ & $\checkmark$ & $\checkmark$ & $\checkmark$ & $\checkmark$ & $\checkmark$ & $\checkmark$ & $\checkmark$ & $\checkmark$ & $x$ & $91 \%$ \\
\hline Zhang et al $(2017)^{41}$ & $\checkmark$ & $\checkmark$ & $\checkmark$ & $\checkmark$ & $\checkmark$ & $\checkmark$ & $\checkmark$ & $\checkmark$ & $\checkmark$ & $\checkmark$ & $\checkmark$ & $100 \%$ \\
\hline Li et al $(2018)^{15}$ & $\checkmark$ & $\checkmark$ & $\checkmark$ & $\checkmark$ & $\checkmark$ & $\checkmark$ & $\checkmark$ & $\checkmark$ & $\checkmark$ & $\checkmark$ & $\checkmark$ & $100 \%$ \\
\hline Mohammed et al $(2018)^{62}$ & $\checkmark$ & $x$ & $\checkmark$ & $\checkmark$ & $x$ & $x$ & $\checkmark$ & $\checkmark$ & $\checkmark$ & $x$ & $x$ & $55 \%$ \\
\hline Mohammed et al $(2018)^{16}$ & $\checkmark$ & $\checkmark$ & $\checkmark$ & $\checkmark$ & $x$ & $x$ & $\checkmark$ & $\checkmark$ & $\checkmark$ & $x$ & $x$ & $64 \%$ \\
\hline Du et al $(2019)^{40}$ & $\checkmark$ & $\checkmark$ & $\checkmark$ & $\checkmark$ & $\checkmark$ & $\checkmark$ & $\checkmark$ & $\checkmark$ & $\checkmark$ & $\checkmark$ & $\checkmark$ & $100 \%$ \\
\hline Jiao et al $(2019)^{66}$ & $x$ & $\checkmark$ & $\checkmark$ & $\checkmark$ & $\checkmark$ & $\checkmark$ & $\checkmark$ & $\checkmark$ & $\checkmark$ & $x$ & $x$ & $73 \%$ \\
\hline Jing et al $(2019)^{17}$ & $\checkmark$ & $\checkmark$ & $\checkmark$ & $\checkmark$ & $\checkmark$ & $x$ & $x$ & $\checkmark$ & $x$ & $x$ & $x$ & $55 \%$ \\
\hline $\mathrm{Li}$ et al $(2019)^{53}$ & $\checkmark$ & $\checkmark$ & $\checkmark$ & $\checkmark$ & $\checkmark$ & $\checkmark$ & $\checkmark$ & $\checkmark$ & $\checkmark$ & $\checkmark$ & $x$ & $91 \%$ \\
\hline Liang et al $(2019)^{52}$ & $\checkmark$ & $\checkmark$ & $\checkmark$ & $\checkmark$ & $\checkmark$ & $\checkmark$ & $\checkmark$ & $\checkmark$ & $\checkmark$ & $\checkmark$ & $\checkmark$ & $100 \%$ \\
\hline Lin et al $(2019)^{51}$ & $\checkmark$ & $\checkmark$ & $\checkmark$ & $\checkmark$ & $\checkmark$ & $\checkmark$ & $\checkmark$ & $\checkmark$ & $\checkmark$ & $\checkmark$ & $\checkmark$ & $100 \%$ \\
\hline Liu et al $(2019)^{65}$ & $\checkmark$ & $\checkmark$ & $\checkmark$ & $\checkmark$ & $\checkmark$ & $\checkmark$ & $\checkmark$ & $\checkmark$ & $x$ & $\checkmark$ & $\checkmark$ & $91 \%$ \\
\hline
\end{tabular}




\begin{tabular}{|c|c|c|c|c|c|c|c|c|c|c|c|c|}
\hline Ma et al $(2019)^{18}$ & $\checkmark$ & $x$ & $\checkmark$ & $\checkmark$ & $x$ & $\checkmark$ & $\checkmark$ & $\checkmark$ & $\checkmark$ & $\checkmark$ & $x$ & $73 \%$ \\
\hline Peng et al $(2019)^{19}$ & $\checkmark$ & $\checkmark$ & $\checkmark$ & $\checkmark$ & $\checkmark$ & $\checkmark$ & $\checkmark$ & $\checkmark$ & $\checkmark$ & $\checkmark$ & $\checkmark$ & $100 \%$ \\
\hline Rehioui et al $(2019)^{20}$ & $\checkmark$ & $x$ & $x$ & $x$ & $\checkmark$ & $\checkmark$ & $x$ & $\checkmark$ & $\checkmark$ & $\checkmark$ & $x$ & $55 \%$ \\
\hline Zou et al $(2019)^{21}$ & $\checkmark$ & $x$ & $\checkmark$ & $\checkmark$ & $x$ & $x$ & $\checkmark$ & $\checkmark$ & $\checkmark$ & $\checkmark$ & $x$ & $64 \%$ \\
\hline Abd Ghani et al $(2020)^{61}$ & $\checkmark$ & $x$ & $\checkmark$ & $x$ & $\checkmark$ & $\checkmark$ & $\checkmark$ & $\checkmark$ & $\checkmark$ & $x$ & $x$ & $64 \%$ \\
\hline Chen et al $(2020)^{68}$ & $\checkmark$ & $\checkmark$ & $\checkmark$ & $\checkmark$ & $\checkmark$ & $\checkmark$ & $\checkmark$ & $\checkmark$ & $\checkmark$ & $\checkmark$ & $x$ & $91 \%$ \\
\hline Chuang et al $(2020)^{23}$ & $\checkmark$ & $\checkmark$ & $x$ & $x$ & $\checkmark$ & $\checkmark$ & $\checkmark$ & $\checkmark$ & $\checkmark$ & $x$ & $x$ & $64 \%$ \\
\hline Cui et al $(2020)^{39}$ & $\checkmark$ & $\checkmark$ & $\checkmark$ & $\checkmark$ & $x$ & $\checkmark$ & $\checkmark$ & $\checkmark$ & $\checkmark$ & $\checkmark$ & $\checkmark$ & $91 \%$ \\
\hline Diao et al $(2020)^{60}$ & $\checkmark$ & $\checkmark$ & $\checkmark$ & $\checkmark$ & $\checkmark$ & $\checkmark$ & $x$ & $\checkmark$ & $\checkmark$ & $\checkmark$ & $\checkmark$ & $91 \%$ \\
\hline Ke et al $(2020)^{49}$ & $\checkmark$ & $\checkmark$ & $\checkmark$ & $\checkmark$ & $\checkmark$ & $\checkmark$ & $\checkmark$ & $\checkmark$ & $\checkmark$ & $\checkmark$ & $\checkmark$ & $100 \%$ \\
\hline Liu et al $(2020)^{38}$ & $\checkmark$ & $\checkmark$ & $\checkmark$ & $\checkmark$ & $x$ & $\checkmark$ & $\checkmark$ & $\checkmark$ & $\checkmark$ & $\checkmark$ & $\checkmark$ & $91 \%$ \\
\hline Men et al $(2020)^{47}$ & $x$ & $\checkmark$ & $\checkmark$ & $\checkmark$ & $x$ & $x$ & $\checkmark$ & $\checkmark$ & $\checkmark$ & $\checkmark$ & $\checkmark$ & $73 \%$ \\
\hline Mohammed et al $(2020)^{26}$ & $\checkmark$ & $x$ & $\checkmark$ & $\checkmark$ & $x$ & $x$ & $\checkmark$ & $\checkmark$ & $x$ & $x$ & $\checkmark$ & $55 \%$ \\
\hline Wang et al $(2020)^{27}$ & $\checkmark$ & $\checkmark$ & $\checkmark$ & $\checkmark$ & $\checkmark$ & $\checkmark$ & $\checkmark$ & $\checkmark$ & $x$ & $x$ & $\checkmark$ & $82 \%$ \\
\hline Wang et al $(2020)^{48}$ & $\checkmark$ & $\checkmark$ & $\checkmark$ & $\checkmark$ & $\checkmark$ & $\checkmark$ & $\checkmark$ & $\checkmark$ & $\checkmark$ & $\checkmark$ & $\checkmark$ & $100 \%$ \\
\hline Xie et al $(2020)^{37}$ & $\checkmark$ & $\checkmark$ & $\checkmark$ & $\checkmark$ & $\checkmark$ & $\checkmark$ & $\checkmark$ & $\checkmark$ & $\checkmark$ & $\checkmark$ & $\checkmark$ & $100 \%$ \\
\hline Xue et al $(2020)^{69}$ & $x$ & $\checkmark$ & $\checkmark$ & $\checkmark$ & $\checkmark$ & $\checkmark$ & $x$ & $\checkmark$ & $\checkmark$ & $\checkmark$ & $\checkmark$ & $82 \%$ \\
\hline Xue et al $(2020)^{46}$ & $\checkmark$ & $x$ & $\checkmark$ & $\checkmark$ & $\checkmark$ & $\checkmark$ & $x$ & $\checkmark$ & $\checkmark$ & $\checkmark$ & $\checkmark$ & $82 \%$ \\
\hline
\end{tabular}


Table 4 (Continued).

\begin{tabular}{|c|c|c|c|c|c|c|c|c|c|c|c|c|}
\hline Studies & Title & Abstract & Rationale & Objectives & $\begin{array}{c}\text { Setting } \\
\text { Description }\end{array}$ & $\begin{array}{l}\text { Problem } \\
\text { Definition }\end{array}$ & $\begin{array}{c}\text { Data } \\
\text { Preparation }\end{array}$ & $\begin{array}{l}\text { Build } \\
\text { Model }\end{array}$ & $\begin{array}{c}\text { Report } \\
\text { Performance }\end{array}$ & $\begin{array}{c}\text { Clinical } \\
\text { Implications }\end{array}$ & Limitations & $\begin{array}{c}\text { Scores } \\
(\%)\end{array}$ \\
\hline Yang, et al $(2020)^{67}$ & $\checkmark$ & $\checkmark$ & $\checkmark$ & $\checkmark$ & $x$ & $\checkmark$ & $\checkmark$ & $\checkmark$ & $\checkmark$ & $\checkmark$ & $x$ & $82 \%$ \\
\hline Zhang et al $(2020)^{58}$ & $\checkmark$ & $\checkmark$ & $\checkmark$ & $\checkmark$ & $\checkmark$ & $\checkmark$ & $\checkmark$ & $\checkmark$ & $\checkmark$ & $\checkmark$ & $\checkmark$ & $100 \%$ \\
\hline Zhang et al $(2020)^{36}$ & $\checkmark$ & $\checkmark$ & $\checkmark$ & $\checkmark$ & $\checkmark$ & $\checkmark$ & $\checkmark$ & $\checkmark$ & $\checkmark$ & $\checkmark$ & $\checkmark$ & $100 \%$ \\
\hline Zhao et al $(2020)^{35}$ & $\checkmark$ & $\checkmark$ & $\checkmark$ & $\checkmark$ & $\checkmark$ & $\checkmark$ & $\checkmark$ & $\checkmark$ & $\checkmark$ & $\checkmark$ & $\checkmark$ & $100 \%$ \\
\hline Zhong et al $(2020)^{29}$ & $\checkmark$ & $\checkmark$ & $\checkmark$ & $\checkmark$ & $\checkmark$ & $\checkmark$ & $\checkmark$ & $\checkmark$ & $\checkmark$ & $\checkmark$ & $\checkmark$ & $100 \%$ \\
\hline Bai et al $(2021)^{30}$ & $\checkmark$ & $x$ & $\checkmark$ & $\checkmark$ & $\checkmark$ & $\checkmark$ & $\checkmark$ & $\checkmark$ & $\checkmark$ & $x$ & $x$ & $73 \%$ \\
\hline Cai et al $(2021)^{31}$ & $x$ & $\checkmark$ & $\checkmark$ & $\checkmark$ & $\checkmark$ & $\checkmark$ & $\checkmark$ & $\checkmark$ & $\checkmark$ & $\checkmark$ & $\checkmark$ & $91 \%$ \\
\hline Tang et al $(2021)^{32}$ & $\checkmark$ & $\checkmark$ & $\checkmark$ & $\checkmark$ & $x$ & $\checkmark$ & $\checkmark$ & $\checkmark$ & $\checkmark$ & $\checkmark$ & $\checkmark$ & $91 \%$ \\
\hline Wen et al $(2021)^{57}$ & $\checkmark$ & $\checkmark$ & $\checkmark$ & $\checkmark$ & $\checkmark$ & $\checkmark$ & $\checkmark$ & $\checkmark$ & $\checkmark$ & $\checkmark$ & $\checkmark$ & $100 \%$ \\
\hline Wong et al $(202 \mathrm{I})^{45}$ & $\checkmark$ & $\checkmark$ & $\checkmark$ & $\checkmark$ & $\checkmark$ & $\checkmark$ & $\checkmark$ & $\checkmark$ & $\checkmark$ & $\checkmark$ & $\checkmark$ & $100 \%$ \\
\hline Wong et al $(2021)^{56}$ & $\checkmark$ & $\checkmark$ & $\checkmark$ & $\checkmark$ & $\checkmark$ & $\checkmark$ & $\checkmark$ & $\checkmark$ & $\checkmark$ & $\checkmark$ & $\checkmark$ & $100 \%$ \\
\hline Wu et al $(202 I)^{34}$ & $\checkmark$ & $\checkmark$ & $\checkmark$ & $\checkmark$ & $\checkmark$ & $\checkmark$ & $\checkmark$ & $\checkmark$ & $\checkmark$ & $x$ & $\checkmark$ & $91 \%$ \\
\hline Zhang et al $(2021)^{33}$ & $\checkmark$ & $\checkmark$ & $\checkmark$ & $\checkmark$ & $\checkmark$ & $\checkmark$ & $\checkmark$ & $\checkmark$ & $\checkmark$ & $\checkmark$ & $\checkmark$ & $100 \%$ \\
\hline
\end{tabular}

Notes: A check mark $(\checkmark)$ refers to passing of the criteria; and a cross mark $(\boldsymbol{X})$ refers to not passing of the criteria. The score refers to the proportion of passed criteria for that publication. Assessment parameters based on the guideline of Luo et al. ${ }^{8}$ 


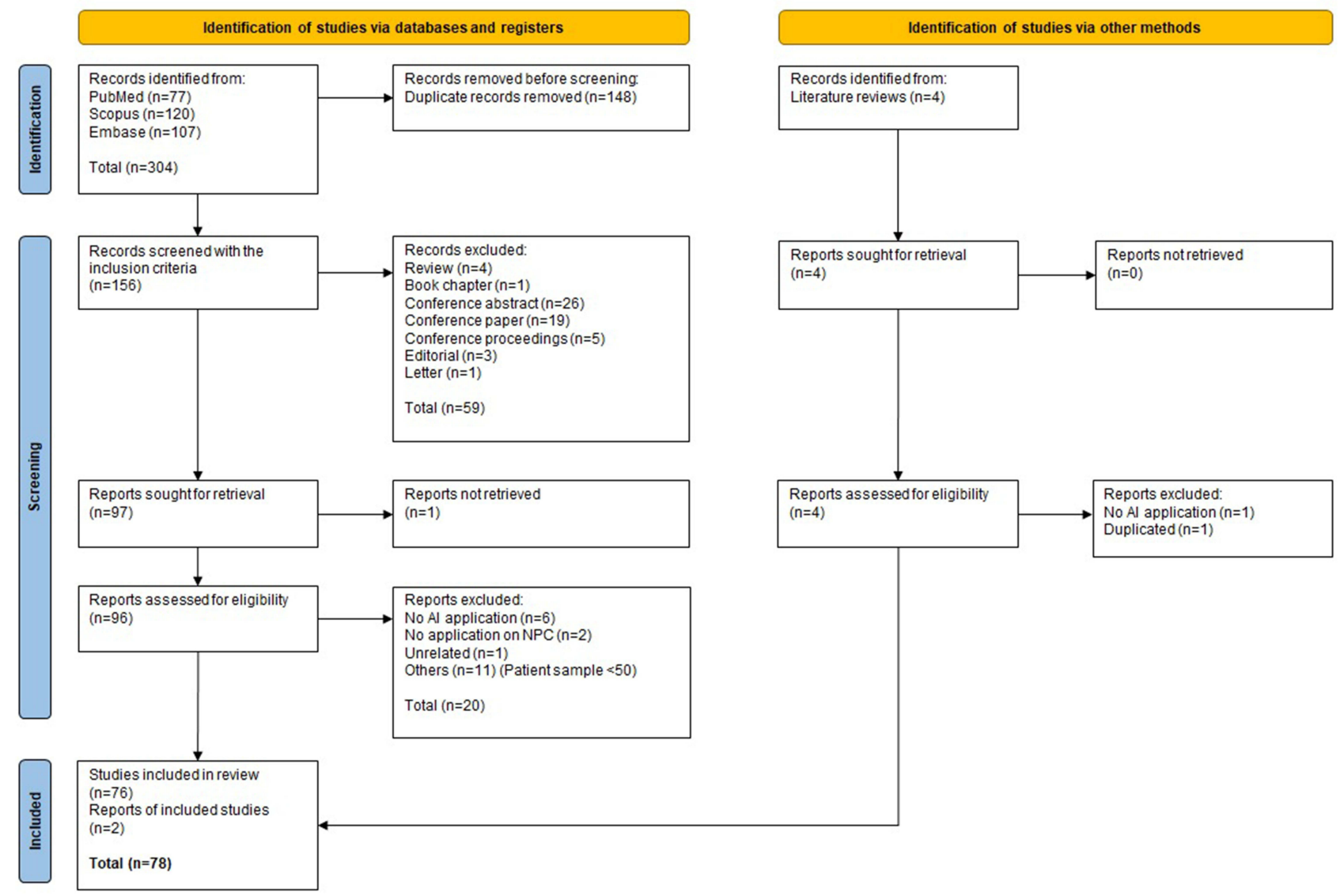

Figure I PRISMA flow diagram 2020.

Notes: Adapted from Page MJ, McKenzie JE, Bossuyt PM, et al.The PRISMA 2020 statement: an updated guideline for reporting systematic reviews. BMJ. 202I;372:n7I. Creative Commons license and disclaimer available from: http://creativecommons.org/licenses/by/4.0/legalcode. ${ }^{6}$

When observing the origins of the studies, 45 were published in Asia, while Morocco and France contributed one study each. Furthermore, 13 papers were collaborated work from multiple countries. The majority of the studies were from the endemic regions.

The articles used various types of data to train the models. $66.7 \%(n=40)$ only used imaging data such as magnetic resonance imaging, computed tomography or endoscopic images. ${ }^{15,16,18,19,21-24,26-28,30,32,34,37-39,41-}$ 43,45-56,58-63,67,69 There were also four studies that included clinicopathological data as well as images for training models, ${ }^{25,31,36,40}$ while three other studies developed models using images, clinicopathological data, and plasma Epstein-Barr virus (EBV) DNA. ${ }^{29,33,35}$ Furthermore, 4 studies used treatment plans, ${ }^{64-66,68}$ while proteins and microRNA expressions data were each extracted by one study. ${ }^{10,44}$ There were also four articles that trained with both clinicopathological and plasma EBV DNA/serology data, ${ }^{12-14,17}$ while one article trained its model with clinicopathological and dosimetric data. ${ }^{57}$
Risk factors ( $\mathrm{n}=2)$, such as demographic, medical history, familial cancer history, dietary, social and environmental factors, were also used to develop AI models. ${ }^{11,20}$

The studies could be categorized into 4 domains, which were auto-contouring $(\mathrm{n}=21),{ }^{15,16,18,22,24,30-32,45-}$ 55,67,69 diagnosis $(\mathrm{n}=17),{ }^{10,15,16,23,26,27,49,52,54,56-63}$ prognosis $(n=20)^{12-14,17,19,25,28,29,33-44}$ and miscellaneous applications $(\mathrm{n}=7),{ }^{11,20,21,64-66,68}$ which included risk factor identification, image registration and radiotherapy planning (Figure 2A). Five studies examined both diagnosis and auto-contouring simultaneously. ${ }^{15,16,49,52,54}$

Analyses on the purpose of the application showed that, only in auto-contouring, DL is the most heavily used (with 19 out of 22 instances). For the rest of the categories (NPC diagnosis, prognosis and miscellaneous applications), ML is the most common technique (more than half of the publications in each category) (Figure 2A). In addition, studies applying DL models selected in this literature review were published from 2017 to 2021, where 

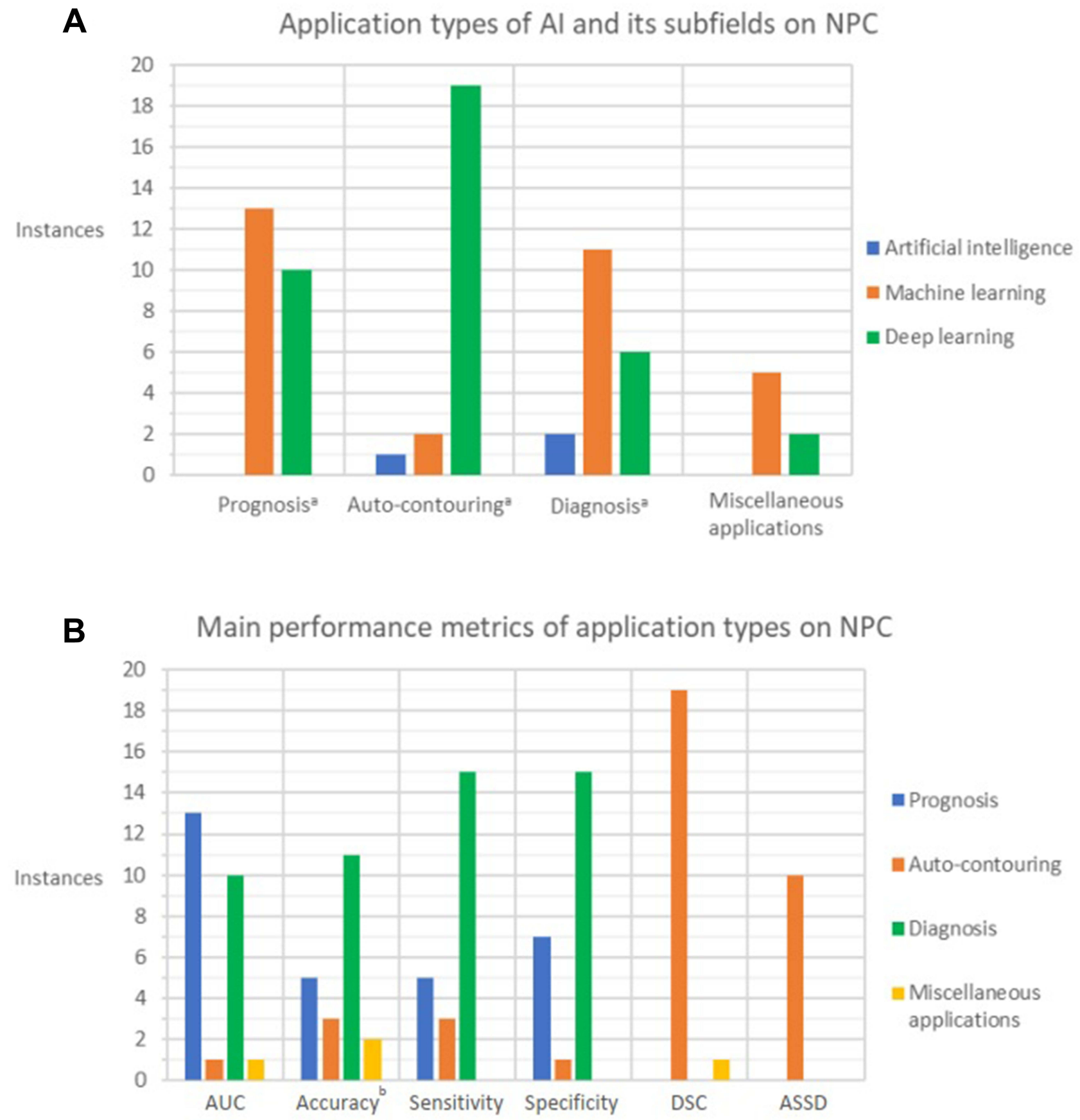

Figure 2 Comparison of studies on Al application for NPC management. (A) Application types of Al and its subfields on NPC; (B) Main performance metrics of application types on NPC.

Notes: ${ }^{a}$ More than one Al subfield (artificial intelligence, machine learning and deep learning) was used in the same study. ${ }^{b}$ Auto-contouring and diagnosis accuracy values were found in the same study. ${ }^{54}$.

Abbreviations: Al, artificial intelligence; AUC, area under the receiver operating characteristic curve; DSC, dice similarity coefficient; ASSD, average symmetric surface distance; NPC, nasopharyngeal carcinoma.

there was a heavier focus on experimenting with DL. It was observed that the majority of the papers applying DL models used various forms of CNN ( $\mathrm{n}=30),{ }^{15,18,19,21-24,28-}$ $34,36,45-53,55,56,60,65,67,69$ while the main ML method used was ANN (n=12). 13,16,26,42-44,54,61-64,68
The primary metrics reported were the area under the receiver operating characteristic curve (AUC), accuracy, sensitivity, specificity, dice similarity coefficient (DSC) and average symmetric surface distance (ASSD), as shown in Figure 2B. 


\section{Performance metric boxplots of Al application types}
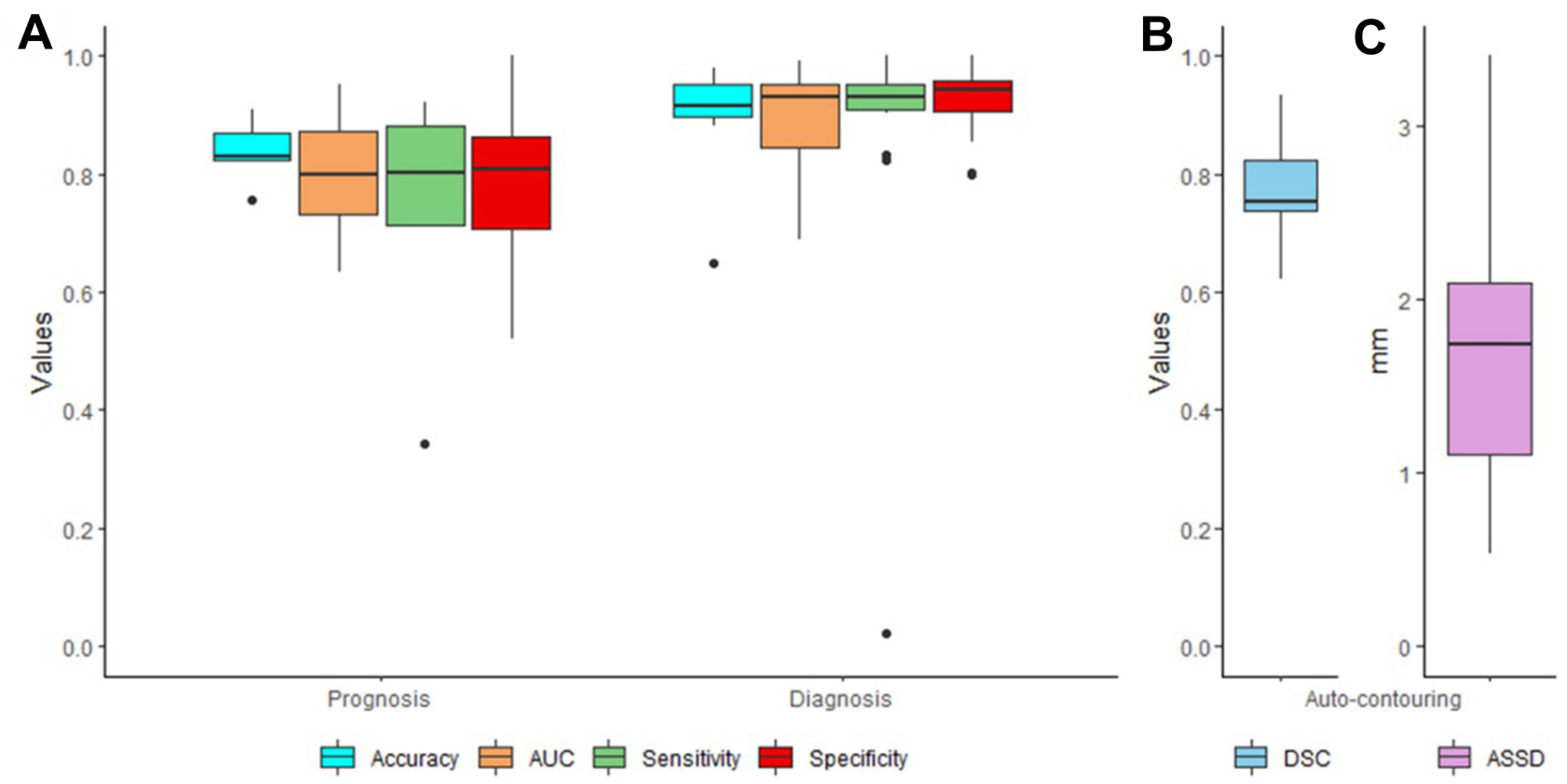

Figure 3 Performance metric boxplots of Al application types on NPC. (A) Prognosis and diagnosis: accuracy, AUC, sensitivity and specificity metric; (B) Auto-contouring: DSC metric; (C) Auto-contouring: ASSD metric.

Abbreviations: Al, artificial intelligence; ASSD, average symmetric surface distance; AUC, area under the receiver operating characteristic curve; DSC, dice similarity coefficient; NPC, nasopharyngeal carcinoma.

AUC was used to evaluate the models' capabilities in 25 papers, with the majority measuring the prognostic $(n=13)^{12-14,19,28,33-35,37,39,40,42,44}$ and diagnostic abilities $(n=10) .{ }^{15,23,26,27,49,56-60}$ Similarly, accuracy was the parameter most frequently reported in the diagnosis and prognosis application: 11 and 5 out of 20 articles respectively. ${ }^{10,12,15,26-28,35,43,44,49,54,56,60-63}$ Sensitivity was the most common studied parameter for diagnostic performance: 15 out of 23 papers.$10,15,16,23,26,27,49,52,54,56,59-63$ The specificity was only reported for prognosis $(n=7)^{12,14,28,34,39,40,43}$ and diagnosis $(\mathrm{n}=15){ }^{10,15,16,23,26,27,49,52,54,56,59-63}$ In addition, the DSC $(\mathrm{n}=20)^{15,18,22,24,30-32,45-53,55,65,67,69}$ and ASSD $(\mathrm{n}=10)-$ $18,22,24,31,32,45,46,48,51,69$ were the primary metrics reported in studies on auto-contouring (Figure 2B).

Performance metrics with five or more instances of each application method were presented in a boxplot (Figure 3). The median AUC, accuracy, sensitivity and specificity of prognosis were $0.8000,0.8300,0.8003$ and 0.8070 respectively, while their range were 0.6330 $0.9510,0.7559-0.9090,0.3440-0.9200$ and $0.5200-1.000$ respectively. For diagnosis, the AUC's median was 0.9300 , while the median accuracy was 0.9150 . In addition, the median sensitivity and specificity were 0.9307 and 0.9413 , respectively. The range for diagnosis' AUC, accuracy, sensitivity and specificity were 0.6900-0.9900, $0.6500-0.9777,0.0215-1.000$ and 0.8000-1.000, respectively. The median DSC value for auto-contouring was 0.7530, while the range was 0.6200-0.9340. Furthermore, the median ASSD for auto-contouring was $1.7350 \mathrm{~mm}$, and the minimum and maximum values found in the studies were $0.5330 \mathrm{~mm}$ and $3.4000 \mathrm{~mm}$, respectively.

\section{Auto-Contouring}

Publications on auto-contouring experimented on segmenting gross tumor volumes, clinical target volume, OARs and primary tumor volumes. The target most delineated was the gross target volume $(n=7),{ }^{30,48,49,51,53,55,69}$ while the second most were the OARs $(n=3) .{ }^{50,52,67}$ The clinical target volumes and the primary tumor volume were studied in two and one articles respectively. ${ }^{46,55,56}$ However, nine articles did not mention the specific target volume contoured. ${ }^{15,16,18,22,24,31,32,47,54}$ Two out of three articles reported that the DSC for delineating optic nerves was substantially lower than the other OARs. ${ }^{52,67}$ In contrast, for the remaining paper, although the segmentation of the optic nerve is not the worst, the paper reported that 
the three OARs it tested, which included optic nerves, were specifically more challenging to contour. ${ }^{50}$ This is because of the low soft-tissue contrast in computed tomography images and their diverse morphological characteristics. When analyzing the OARs, automatic delineation of the eyes yielded the best DSC. Furthermore, apart from the spinal cord, optic nerve and optic chiasm, the AI models have a DSC value greater than 0.8 when contouring OARs. ${ }^{50,52,67}$

\section{Diagnosis}

As for the detection of NPC, six papers compared the performance of AI and humans. Two of them found that AIs had better diagnostic capabilities than humans (oncologists and experienced radiologists), ${ }^{15,49}$ while another two reported that AIs had similar performances to ear, nose and throat specialists. ${ }^{16,62}$ However, the last two papers found that it depends on the experience of the person. For example, senior-level clinicians performed better than the AI, while junior level ones were worse. ${ }^{23,60}$ This is because of the variations in possible sizes, shapes, locations, and image intensities of NPC, making it difficult to determine the diagnosis. These factors make it challenging for clinicians with less experience, and it showed that AI diagnostic tools could support junior-level clinicians.

On the other hand, within the 17 papers experimenting on the diagnostic application of AI, three articles analyzed radiation-induced injury diagnosis. ${ }^{27,57,58}$ Two of which were concerned with radiation-induced temporal lobe injury, ${ }^{57,58}$ while the remaining one predicted the fibrosis level of neck muscles after radiotherapy. ${ }^{27}$ It was suggested that through early detection and prediction of radiation-induced injuries, preventive measures could be taken to minimize the side effects.

\section{Prognosis}

For studies on NPC prognosis, 11 out of 20 publications focused on predicting treatment outcomes, with the majority including disease-free survival as one of the study objectives. ${ }^{12,13,17,19,29,33,36,39-42}$ The rest studied treatment response prediction $(\mathrm{n}=2),{ }^{35,43}$ predicting patients' risk of survival $(\mathrm{n}=5),{ }^{14,25,37,38,44} \mathrm{~T}$ staging prediction and the prediction of distant metastasis $(n=2) .{ }^{28,34}$ Therefore, the versatility of AI in different functionalities was demonstrated. The performances of the models were reported in (Table 1) and the main metric analyzed was AUC with 13 out of 25 articles (Figure 2B).

\section{Miscellaneous Applications}

In addition to the above aspects, AI was also used to study risk factor identification $(\mathrm{n}=2),{ }^{11,20}$ image registration $(\mathrm{n}=1)^{21}$ and dose/dose-volume histogram (DVH) distribution $(n=4) .{ }^{64-66,68}$ In particular, dose/DVH distribution prediction was frequently used for treatment planning. A better understanding of the doses given to the target and OARs can help clinicians give a more individualized treatment plan with better consistency and a lower planning duration. However, further development is required to obtain similar plan qualities as created by people. This is because one paper's model showed the same quality as manual planning by an experienced physicist, ${ }^{64}$ but another study using a different model was unable to achieve a similar plan quality designed by even a junior physicist. $^{68}$

\section{Discussion}

As evident in this systematic review, there is an exponential growth in interest to apply AI for the clinical management of NPC. A large proportion of the articles collected were published from 2019 to $2021(\mathrm{n}=45)$ compared to that from 2010 to $2018(\mathrm{n}=15)$.

A heavier focus is also placed on specific fields of AIs, such as ML and DL. There are only three reports on AI, while there are 31 studies on ML and 37 on DL. The choice of AI subfield sometimes depends on the task. For example, $86 \%$ of the papers focused on DL for NPC autocontouring $(n=19)$, while although the majority of the studies in the other applications used ML, they were more evenly distributed (Figure 2A). The reason why there is such a significant difference in the type of AI used in auto-contouring may be due to the capability of the algorithms and the nature of the data. The medical images acquired have many factors affecting the autocontouring quality; these include the varying tumour sizes and shapes, image resolution, contrast between regions, noise and lack of consistency during data acquisition being collected from different institutions. ${ }^{70}$ Because of these challenges, ML-based algorithms have difficulty in performing automated segmentation on NPC as image processing before training is required, which is time-consuming. Furthermore, handcrafted features are necessary to precisely contour each organ or tumour as there are significant variations in size and shape for NPC. On the other hand, DL does not have this issue as they can 
process the raw data directly without the need for handcrafted features. $^{70}$

ANN is the backbone of DL, as DL algorithms are ANNs with multiple ( 2 or more) hidden layers. In the development of AI applications for NPC, $80 \%$ of the studied articles incorporated either ANN or DL technique in their models$12,13,15-19,21-26,28-34,36,38,39,42-56,60-69$ because neural networks are generally better for image recognition. However, one study cautioned that ANNs were not necessarily better than other ML models in NPC identification. ${ }^{61}$ Hence, even though DL-based models and ANNs should be considered the primary development focus, other ML techniques should still not be neglected.

Based on the literature collected, the integration of AI applications in each category is beneficial to the practitioner. Automated contouring by AIs not only can make contouring less time-consuming for clinicians, ${ }^{46,51,53,64}$ it can also help to improve the user's accuracy. ${ }^{51}$ Similarly, AI can be used to reduce the treatment planning time for radiotherapy ${ }^{64}$ thus improve the efficiency and effectiveness of the radiotherapy planning process.

For some NPC studies, additional features from images and parameters were extracted to further improve the performances of models. However, it should be noted that not all features are suitable as some features have a more significant impact on the model's performance than others. $^{40,57,58,61}$ Therefore, feature selection should be considered where possible.

At its current state, AI cannot yet replace humans to perform the most complex and time-consuming tasks. This is because multiple articles which compared the performance of their developed model with medical professionals showed conflicting results. The reason for this is that the experience of the clinician is an important factor that affects the resulting comparison. The models developed by Chuang et al and Diao et al performed better than junior-level professionals, but performed worse when compared to more experienced clinicians. ${ }^{23,60}$ One article even showed that an AI model had a lower capability than a junior physicist. ${ }^{68}$ Furthermore, the quality of the training data and the experience of the AI developers are critical.

The review revealed that $\mathrm{AI}$ at its current state still has several limitations. The first concern was the uncertainty regarding the generalizability of the models, because datasets of many studies are retrospective and single institutional in nature. ${ }^{15,19,28,33,35-38,41,48,57-59}$ The dataset may not represent the true population and may only represent a population subgroup or a region. Hence, this reduces the applicability of the models and affects their performance when applied to other datasets. Another reason was the difference in scan protocol between institutions. Variations in tissue contrasts or field of views may affect the performance as the model was not trained for the same condition. ${ }^{45,56}$ Therefore, consistency of scan protocols among different institutions is important to facilitate AI model training and validation.

Another limitation was the small amount of data used to train the models. $33 \%(n=20)$ of the articles chosen had $\leq 150$ total samples for both training and testing the model. The reason for this was not only were the articles usually based on single-centre data, but also because NPC is less common compared to other cancers. This particularly affects DL-based models as they are more reliant on a much larger dataset to achieve their potential when compared to ML models; over-fitting will likely occur when there is only limited data; thus, data augmentation is often used to increase the dataset size. In addition, some studies had patient selection bias, while others had concerns about not implementing multi-modality inputs into the training model (Table 1).

Future work should address these issues when developing new models. Possible solutions include incorporating other datasets or cooperating with other institutions for external validation or to expand the dataset, which were lacking in most of the analysed papers in this review. The former suggestion can boost generalizability and avoid any patient selection bias, while the latter method can increase the capability of the AI models by providing more training samples. Other methods to expand dataset have also been explored, one of which is by using big data which can be done at a much larger scale. Big data can be defined as the vast data generated by technology and the internet of things, allowing easier access to information. ${ }^{71}$ In the healthcare sector, it will allow easier access to an abundance of medical data which will facilitate AI model training. However, with the large collection of data, privacy protection becomes a serious challenge. Therefore, future studies are required to investigate how to implement it.

The performances of the AI models could also be improved by increasing the amount of data and diversifying it with data augmentation techniques which were performed in some of the studies. However, it should be noted that with an increase in training samples, more data labelling will be required, making the process more time-consuming. Hence, one study proposed the use of continual 
learning, which it found to boost the model's performance while reducing the labelling effort. ${ }^{47}$ However, continual learning is susceptible to catastrophic forgetting, which is a long-standing and highly challenging issue. ${ }^{72}$ Thus, further investigation into methods to resolve this problem would be required to make it easier to implement in other research settings.

There are several limitations in this literature review. The metric performance results extracted from the publications were insufficient to perform a meta-analysis. Hence, the insight obtained from this review is not comprehensive enough. The quality of the included studies was also not consistent, which may affect the analysis performed.

\section{Conclusion}

There is growing evidence that AI can be applied in various situations, particularly as a supporting tool in prognostic, diagnostic and auto-contouring applications and to provide patients with a more individualized treatment plan. DL-based algorithm was found to be the most frequently used AI subfield and usually obtained good results when compared to other methods. However, limited dataset and generalizability are key challenges that need to be overcome to further improve the performances and accessibility of AI models. Nevertheless, studies on AI demonstrated highly promising potential in supporting medical professionals in the management of NPC; therefore, more concerted efforts in swift development is warranted.

\section{Disclosure}

Dr Nabil F Saba reports personal fees from Merck, GSk, Pfizer, Uptodate, and Springer, outside the submitted work; and Research funding from BMS and Exelixis. Professor Raymond KY Tsang reports non-financial support from Atos Medical Inc., outside the submitted work. The authors report no other conflicts of interest in this work.

\section{References}

1. Sung H, Ferlay J, Siegel RL, et al. Global cancer statistics 2020: GLOBOCAN estimates of incidence and mortality worldwide for 36 cancers in 185 countries. CA Cancer J Clin. 2021;71(3):209-249. doi:10.3322/caac. 21660

2. Ferlay J, Ervik M, Lam F, et al. Global cancer observatory: cancer today; 2020. Available from: https://gco.iarc.fr/today. Accessed June 4, 2021.
3. Lee AWM, Ma BBY, Ng WT, Chan ATC. Management of nasopharyngeal carcinoma: current practice and future perspective. $J$ Clin Oncol. 2015;33(29):3356-3364. doi:10.1200/JCO.2015.60.9347

4. Chan JW, Parvathaneni U, Yom SS. Reducing radiation-related morbidity in the treatment of nasopharyngeal carcinoma. Future Oncology. 2017;13(5):425-431. doi:10.2217/fon-20160410

5. Shimizu H, Nakayama KI. Artificial intelligence in oncology. Cancer Sci. 2020;111(5):1452-1460. doi:10.1111/cas.14377

6. Page MJ, McKenzie JE, Bossuyt PM, et al. The PRISMA 2020 statement: an updated guideline for reporting systematic reviews. BMJ. 2021;372:n71. doi:10.1136/bmj.n71

7. Whiting PF, Rutjes AWS, Westwood ME. QUADAS-2: a revised tool for the quality assessment of diagnostic accuracy studies. Ann Intern Med. 2011;155(8):529-536. doi:10.7326/0003-4819-155-8201110180-00009

8. Luo W, Phung D, Tran T, et al. Guidelines for developing and reporting machine learning predictive models in biomedical research: a multidisciplinary view. $J$ Med Internet Res. 2016;18(12):e323. doi:10.2196/jmir.5870

9. Alabi RO, Youssef O, Pirinen M, et al. Machine learning in oral squamous cell carcinoma: current status, clinical concerns and prospects for future-A systematic review. Artif Intell Med. 2021;115:102060. doi:10.1016/j.artmed.2021.102060

10. Wang HQ, Zhu HL, Cho WCS, Yip TTC, Ngan RKC, Law SCK. Method of regulatory network that can explore protein regulations for disease classification. Artif Intell Med. 2010;48(2):119-127. doi:10.1016/j.artmed.2009.07.011

11. Aussem A, de Morais SR, Corbex M. Analysis of nasopharyngeal carcinoma risk factors with Bayesian networks. Artif Intell Med. 2012;54(1):53-62. doi:10.1016/j.artmed.2011.09.002

12. Kumdee O, Bhongmakapat T, Ritthipravat P. Prediction of nasopharyngeal carcinoma recurrence by neuro-fuzzy techniques. Fuzzy Sets Syst. 2012;203:95-111. doi:10.1016/j.fss.2012.03.004

13. Ritthipravat $\mathrm{P}$, Kumdee O, Bhongmakap T. Efficient missing data technique for prediction of nasopharyngeal carcinoma recurrence. Inf Technol J. 2013;12:1125-1133. doi:10.3923/ itj.2013.1125.1133

14. Jiang R, You R, Pei X-Q, et al. Development of a ten-signature classifier using a support vector machine integrated approach to subdivide the M1 stage into M1a and M1b stages of nasopharyngeal carcinoma with synchronous metastases to better predict patients' survival. Oncotarget. 2016;7(3):3645-3657. doi:10.18632/onco target. 6436

15. Li C, Jing B, Ke L, et al. Development and validation of an endoscopic images-based deep learning model for detection with nasopharyngeal malignancies. Cancer Commun. 2018;38(1):59. doi:10.1186/s40880-018-0325-9

16. Mohammed MA, Abd Ghani MK, Arunkumar N, Mostafa SA, Abdullah MK, Burhanuddin MA. Trainable model for segmenting and identifying Nasopharyngeal carcinoma. Comput Electr Eng. 2018;71:372-387. doi:10.1016/j.compeleceng.2018.07.044

17. Jing $B$, Zhang $T$, Wang $Z$, et al. A deep survival analysis method based on ranking. Artif Intell Med. 2019;98:1-9. doi:10.1016/j. artmed.2019.06.001

18. Ma Z, Zhou S, Wu X, et al. Nasopharyngeal carcinoma segmentation based on enhanced convolutional neural networks using multi-modal metric learning. Phys Med Biol. 2019;64(2):025005. doi:10.1088/ 1361-6560/aaf5da

19. Peng H, Dong D, Fang M-J, et al. Prognostic value of deep learning PET/CT-based radiomics: potential role for future individual induction chemotherapy in advanced nasopharyngeal carcinoma. Clin Cancer Res. 2019;25(14):4271-4279. doi:10.1158/1078-0432.CCR18-3065

20. Rehioui $\mathrm{H}$, Idrissi A. On the use of clustering algorithms in medical domain. Int J Artifi Intell. 2019;17:236. 
21. Zou M, Hu J, Zhang H, et al. Rigid medical image registration using learning-based interest points and features. Comput Mater Continua. 2019;60(2):511-525. doi:10.32604/cmc.2019.05912

22. Chen H, Qi Y, Yin Y, et al. MMFNet: a multi-modality MRI fusion network for segmentation of nasopharyngeal carcinoma. Neuroc omputing. 2020;394:27-40. doi:10.1016/j.neucom.2020.02.002

23. Chuang W-Y, Chang S-H, Yu W-H, et al. Successful identification of nasopharyngeal carcinoma in nasopharyngeal biopsies using deep learning. Cancers (Basel). 2020;12(2):507. doi:10.3390/cancers12020507

24. Guo F, Shi C, Li X, Wu X, Zhou J, Lv J. Image segmentation of nasopharyngeal carcinoma using $3 \mathrm{D} \mathrm{CNN}$ with long-range skip connection and multi-scale feature pyramid. Soft Comput. 2020;24 (16):12671-12680. doi:10.1007/s00500-020-04708-y

25. Jing B, Deng Y, Zhang T, et al. Deep learning for risk prediction in patients with nasopharyngeal carcinoma using multi-parametric MRIs. Comput Methods Programs Biomed. 2020;197:105684. doi:10.1016/j.cmpb.2020.105684

26. Mohammed MA, Abd Ghani MK, Arunkumar N, et al. Decision support system for nasopharyngeal carcinoma discrimination from endoscopic images using artificial neural network. J Supercomput. 2020;76(2):1086-1104. doi:10.1007/s11227-018-2587-z

27. Wang J, Liu R, Zhao Y, et al. A predictive model of radiation-related fibrosis based on the radiomic features of magnetic resonance imaging and computed tomography. Transl Cancer Res. 2020;9(8):47264738. doi:10.21037/tcr-20-751

28. Yang Q, Guo Y, Ou X, Wang J, Hu C. Automatic T staging using weakly supervised deep learning for nasopharyngeal carcinoma on MR images. Journal of Magnetic Resonance Imaging. 2020;52 (4):1074-1082. doi:10.1002/jmri.27202

29. Zhong L-Z, Fang X-L, Dong D, et al. A deep learning MR-based radiomic nomogram may predict survival for nasopharyngeal carcinoma patients with stage T3N1M0. Radiother Oncol. 2020;151:1-9. doi:10.1016/j.radonc.2020.06.050

30. Bai X, Hu Y, Gong G, Yin Y, Xia Y. A deep learning approach to segmentation of nasopharyngeal carcinoma using computed tomography. Biomed Signal Process. 2021;64:102246. doi:10.1016/j. bspc. 2020.102246

31. Cai M, Wang J, Yang Q, et al. Combining images and t-staging information to improve the automatic segmentation of nasopharyngeal carcinoma tumors in MR images. IEEE Access. 2021;9:2132321331. doi:10.1109/ACCESS.2021.3056130

32. Tang P, Zu C, Hong M, et al. DA-DSUnet: dual attention-based dense SU-net for automatic head-and-neck tumor segmentation in MRI images. Neurocomputing. 2021;435:103-113. doi:10.1016/j. neucom.2020.12.085

33. Zhang $\mathrm{L}$, Wu X, Liu J, et al. MRI-based deep-learning model for distant metastasis-free survival in locoregionally advanced Nasopharyngeal carcinoma. J Magn Reson Imaging. 2021;53 (1):167-178. doi:10.1002/jmri.27308

34. Wu X, Dong D, Zhang L, et al. Exploring the predictive value of additional peritumoral regions based on deep learning and radiomics: a multicenter study. Med Phys. 2021;48(5):2374-2385. doi:10.1002/mp.14767

35. Zhao L, Gong J, Xi Y, et al. MRI-based radiomics nomogram may predict the response to induction chemotherapy and survival in locally advanced nasopharyngeal carcinoma. Eur Radiol. 2020;30 (1):537-546. doi:10.1007/s00330-019-06211-x

36. Zhang F, Zhong L-Z, Zhao X, et al. A deep-learning-based prognostic nomogram integrating microscopic digital pathology and macroscopic magnetic resonance images in nasopharyngeal carcinoma: a multi-cohort study. Ther Adv Med Oncol. 2020;12:1758 835920971416. doi:10.1177/1758835920971416

37. Xie C, Du R, Ho JWK, et al. Effect of machine learning re-sampling techniques for imbalanced datasets in 18F-FDG PET-based radiomics model on prognostication performance in cohorts of head and neck cancer patients. Eur J Nucl Med Mol Imaging. 2020;47(12):28262835. doi:10.1007/s00259-020-04756-4
38. Liu K, Xia W, Qiang M, et al. Deep learning pathological microscopic features in endemic nasopharyngeal cancer: prognostic value and protentional role for individual induction chemotherapy. Cancer Med. 2020;9(4):1298-1306. doi:10.1002/cam4.2802

39. Cui C, Wang S, Zhou J, et al. Machine learning analysis of image data based on detailed MR image reports for nasopharyngeal carcinoma prognosis. Biomed Res Int. 2020;2020:8068913. doi:10.1155/ 2020/8068913

40. Du R, Lee VH, Yuan H, et al. Radiomics model to predict early progression of nonmetastatic nasopharyngeal carcinoma after intensity modulation radiation therapy: a multicenter study. Radiology. 2019;1(4):e180075. doi:10.1148/ryai.2019180075

41. Zhang B, Tian J, Dong D, et al. Radiomics features of multiparametric MRI as novel prognostic factors in advanced nasopharyngeal carcinoma. Clin Cancer Res. 2017;23(15):4259-4269. doi:10.1158/ 1078-0432.CCR-16-2910

42. Zhang B, He X, Ouyang F, et al. Radiomic machine-learning classifiers for prognostic biomarkers of advanced nasopharyngeal carcinoma. Cancer Lett. 2017;403:21-27. doi:10.1016/j.canlet.2 017.06 .004

43. Liu J, Mao Y, Li Z, et al. Use of texture analysis based on contrastenhanced MRI to predict treatment response to chemoradiotherapy in nasopharyngeal carcinoma. J Magn Reson Imaging. 2016;44(2):445455.

44. Zhu W, Kan X, Calogero RA. Neural network cascade optimizes MicroRNA biomarker selection for nasopharyngeal cancer prognosis. PLoS One. 2014;9(10):e110537. doi:10.1371/journal.pone.0110537

45. Wong LM, Ai QYH, Mo FKF, Poon DMC, King AD. Convolutional neural network in nasopharyngeal carcinoma: how good is automatic delineation for primary tumor on a non-contrast-enhanced fat-suppressed T2-weighted MRI? Jpn J Radiol. 2021;39(6):571-579. doi:10.1007/s11604-021-01092-x

46. Xue X, Qin N, Hao X, et al. Sequential and iterative auto-segmentation of high-risk clinical target volume for radiotherapy of nasopharyngeal carcinoma in planning CT images. Front Oncol. 2020;10:1134. doi:10.3389/fonc.2020.01134

47. Men K, Chen X, Zhu J, et al. Continual improvement of nasopharyngeal carcinoma segmentation with less labeling effort. Phys Med. 2020;80:347-351. doi:10.1016/j.ejmp.2020.11.005

48. Wang X, Yang G, Zhang Y, et al. Automated delineation of nasopharynx gross tumor volume for nasopharyngeal carcinoma by plain CT combining contrast-enhanced CT using deep learning. J Radiat Res Appl Sci. 2020;13(1):568-577. doi:10.1080/16878507.2020.1795565

49. Ke L, Deng Y, Xia W, et al. Development of a self-constrained 3D DenseNet model in automatic detection and segmentation of nasopharyngeal carcinoma using magnetic resonance images. Oral Oncol. 2020;110:104862. doi:10.1016/j.oraloncology.2020.104862

50. Zhong T, Huang X, Tang F, Liang S, Deng X, Zhang Y. Boostingbased cascaded convolutional neural networks for the segmentation of CT organs-at-risk in nasopharyngeal carcinoma. Med Phys. 2019;46(12):5602-5611. doi:10.1002/mp.13825

51. Lin L, Dou Q, Jin Y-M, et al. Deep learning for automated contouring of primary tumor volumes by MRI for nasopharyngeal carcinoma. Radiology. 2019;291(3):677-686. doi:10.1148/radiol.2019182012

52. Liang S, Tang F, Huang X, et al. Deep-learning-based detection and segmentation of organs at risk in nasopharyngeal carcinoma computed tomographic images for radiotherapy planning. Eur Radiol. 2019;29(4):1961-1967. doi:10.1007/s00330-018-5748-9

53. Li S, Xiao J, He L, Peng X, Yuan X. The tumor target segmentation of nasopharyngeal cancer in CT images based on deep learning methods. Technol Cancer Res Treat. 2019;18:1533033819884561. doi: $10.1177 / 1533033819884561$

54. Mohammed MA, Abd Ghani MK, Hamed RI, Ibrahim DA, Abdullah MK. Artificial neural networks for automatic segmentation and identification of nasopharyngeal carcinoma. J Comput Sci. 2017;21:263274. 
55. Men K, Chen X, Zhang Y, et al. Deep deconvolutional neural network for target segmentation of nasopharyngeal cancer in planning computed tomography images. Front Oncol. 2017;7:315. doi:10.3389/fonc. 2017.00315

56. Wong LM, King AD, Ai QYH, et al. Convolutional neural network for discriminating nasopharyngeal carcinoma and benign hyperplasia on MRI. Eur Radiol. 2021;31(6):3856-3863. doi:10.1007/s00330020-07451-y

57. Wen D-W, Lin L, Mao Y-P, et al. Normal tissue complication probability (NTCP) models for predicting temporal lobe injury after intensity-modulated radiotherapy in nasopharyngeal carcinoma: a large registry-based retrospective study from China. Radiother Oncol. 2021;157:99-105. doi:10.1016/j.radonc.2021.01.008

58. Zhang B, Lian Z, Zhong L, et al. Machine-learning based MRI radiomics models for early detection of radiation-induced brain injury in nasopharyngeal carcinoma. BMC Cancer. 2020;20(1):502. doi:10.1186/s12885-020-06957-4

59. Du D, Feng H, Lv W, et al. Machine learning methods for optimal radiomics-based differentiation between recurrence and inflammation: application to nasopharyngeal carcinoma post-therapy PET/CT images. Mol Imaging Biol. 2020;22(3):730-738. doi:10.1007/s11307019-01411-9

60. Diao S, Hou J, Yu H, et al. Computer-aided pathologic diagnosis of nasopharyngeal carcinoma based on deep learning. Am J Pathol. 2020;190(8):1691-1700. doi:10.1016/j.ajpath.2020.04.008

61. Abd Ghani MK, Mohammed MA, Arunkumar N, et al. Decisionlevel fusion scheme for nasopharyngeal carcinoma identification using machine learning techniques. Neural Comput Appl. 2020;32 (3):625-638. doi:10.1007/s00521-018-3882-6

62. Mohammed MA, Abd Ghani MK, Arunkumar N, Hamed RI, Abdullah MK, Burhanuddin MA. A real time computer aided object detection of nasopharyngeal carcinoma using genetic algorithm and artificial neural network based on Haar feature fear. Future Gener Comput Syst. 2018;89:539-547. doi:10.1016/j.future.2018.07.022
63. Wang Y-W, Wu C-S, Zhang G-Y, et al. Can parameters other than minimal axial diameter in MRI and PET/CT further improve diagnostic accuracy for equivocal retropharyngeal lymph nodes in nasopharyngeal carcinoma? PLoS One. 2016;11(10):e0163741-e0163741. doi:10.1371/journal.pone.0163741

64. Bai P, Weng X, Quan K, et al. A knowledge-based intensity-modulated radiation therapy treatment planning technique for locally advanced nasopharyngeal carcinoma radiotherapy. Radiat Oncol. 2020;15(1):188. doi:10.1186/s13014-020-01626-Z

65. Liu Z, Fan J, Li M, et al. A deep learning method for prediction of three-dimensional dose distribution of helical tomotherapy. Med Phys. 2019;46(5):1972-1983. doi:10.1002/mp.13490

66. Jiao S-X, Chen L-X, Zhu J-H, Wang M-L, Liu X-W. Prediction of dose-volume histograms in nasopharyngeal cancer IMRT using geometric and dosimetric information. Phys Med Biol. 2019;64 (23):23NT04. doi:10.1088/1361-6560/ab50eb

67. Yang X, Li X, Zhang X, Song F, Huang S, Xia Y. Segmentation of organs at risk in nasopharyngeal cancer for radiotherapy using a selfadaptive Unet network. Nan Fang Yi Ke Da Xue Xue Bao. 2020;40 (11):1579-1586. doi:10.12122/j.issn.1673-4254.2020.11.07

68. Chen X, Yang J, Yi J, Dai J. Quality control of VMAT planning using artificial neural network models for nasopharyngeal carcinoma. Chin J Radiol Med Prot. 2020;40(2):99-105.

69. Xue X, Hao X, Shi J, Ding Y, Wei W, An H. Auto-segmentation of high-risk primary tumor gross target volume for the radiotherapy of nasopharyngeal carcinoma. J Image Graph. 2020;25(10):2151-2158.

70. Rizwan I, Haque I, Neubert J. Deep learning approaches to biomedical image segmentation. Inf Med Unlocked. 2020;18:100297. doi:10.1016/j.imu.2020.100297

71. Leclerc B, Cale J. Big Data. Milton, UK: Taylor \& Francis Group; 2020.

72. Parisi GI, Kemker R, Part JL, Kanan C, Wermter S. Continual lifelong learning with neural networks: a review. Neural Netw. 2019;113:54-71. doi:10.1016/j.neunet.2019.01.012

\section{Publish your work in this journal}

Cancer Management and Research is an international, peer-reviewed open access journal focusing on cancer research and the optimal use of preventative and integrated treatment interventions to achieve improved outcomes, enhanced survival and quality of life for the cancer patient.
The manuscript management system is completely online and includes a very quick and fair peer-review system, which is all easy to use. Visit http://www.dovepress.com/testimonials.php to read real quotes from published authors. 